\title{
INTEGRAL REPRESENTATION OF FUNCTIONS AND DISTRIBUTIONS POSITIVE DEFINITE RELATIVE TO THE ORTHOGONAL GROUP
}

\author{
BY
}

\section{A. EDWARD NUSSBAUM( $\left.{ }^{1}\right)$}

ABSTRACT. A continuous function $f$ on an open ball $B$ in $R^{N}$ is called positive definite relative to the orthogonal group $O(N)$ if $f$ is radial and $\iint f(x-y) \phi(x) \overline{\phi(y)} d x d y \geq 0$ for all radial $\phi \in C_{0}^{\infty}(B / 2)$. It is shown that $f$ is positive definite in $B$ relative to $O(N)$ if and only if $f$ has an integral representation $f(x)=\int e^{i x \cdot t} d \mu_{1}(t)+\int e^{x \cdot t} d \mu_{2}(t)$, where $\mu_{1}$ and $\mu_{2}$ are bounded, positive, rotation invariant Radon measures on $R^{N}$ and $\mu_{2}$ may be taken to be zero if, in addition to $f$ being positive definite relative to $O(N), \iint f(x-y)(-\Delta \phi)(x) \overline{\phi(y)} d x d y$ $\geq 0$ for all radial $\phi \in C_{0}^{\infty}(B / 2)$. Both conditions are satisfied if $f$ is a radial positive definite function in $B$. Thus the theorem yields as a special case Rudin's theorem on the extension of radial positive definite functions. The result is extended further to distributions.

1. Introduction. Let $N$ be a fixed positive integer. We denote by $R^{N}$ the $N$-dimensional Euclidean space. $|x|$ denotes the usual norm: If $x=\left(x_{1}, x_{2}\right.$, $\left.\cdots, x_{N}\right) \in R^{N}$ and $y=\left(y_{1}, y_{2}, \ldots, y_{N}\right) \in R^{N}, x \cdot y=\Sigma x_{i} y_{i}$ and $|x|=(x \cdot x)^{1 / 2}$.

Suppose now that $G$ is an open symmetric neighborhood of the origin in $R^{n}$ (i.e. $x \in G$ implies that $-x \in G$ ) and $f$ a complex valued function defined on $G$. $f$ is said to be positive definite in $G$ if

$$
\sum_{i=1}^{n} \sum_{j=1}^{n} \alpha_{i} \bar{\alpha}_{j} f\left(x_{i}-x_{j}\right) \geq 0
$$

for all choices of complex numbers $\alpha_{1}, \alpha_{2}, \ldots, \alpha_{n}$ and points $x_{1}, x_{2}, \ldots, x_{n}$ in $G / 2=\left\{x \in R^{N} \mid 2 x \in G\right\}$.

For a continuous complex valued function $f$ defined on $G,(1)$ is clearly equivalent to

$$
\int_{G / 2} \int_{G / 2} f(x-y) \phi(x) \overline{\phi(y)} d x d y \geq 0 \text { for all } \phi \in C_{0}^{\infty}(G / 2) \text {. }
$$

$C_{0}^{\infty}(G / 2)$ denotes the vector space of all complex valued infinitely differentiable functions in $G / 2$ with compact support.

Received by the editors September 24, 1971 . $46 \mathrm{~F} 05$.

AMS (MOS) subject classifications (1970). Primary 42A88, 43A35, 47A70; Secondary

Key words and phrases. Positive definite functions, positive definite distributions, expansions into generalized eigenvectors, nuclear spectral theorem.

(1) This work was in part supported by National Science Foundation Grant NSFGP-19588. 
Does every continuous positive definite function $f$ in $G$ bave an extension to $R^{N}$ which is positive definite in $R^{N}$ ?

If $N=1$ and $G$ is an interval the answer is yes. The result is due to Krein [11].

If $N \geq 2$ and $G$ is an $N$-dimensional cube the answer is in general no. This negative result was first proved by Calderon and Pepinsky [3] for the group of lattice points in $R^{N}$ and then extended by Rudin [17] to $R^{N}$. For further results on this extension problem of positive definite functions we refer the reader to the papers by Devinatz [4] and Eskin [5] and the monograph by Berezanskii [1].

Recently W. Rudin [18] has proved that if $G$ is a ball in $R^{N}$ and $f$ is a continuous radial positive definite function in $G$ then the answer to the extension problem is affirmative; there exists a radial positive definite function $F$ in $R^{n}$ such that $F(x)=f(x)$ for all $x \in G$. (Note. If a positive definite function is continuous at the origin it is continuous in its domain. Thus $F$ is necessarily continuous.) It follows from this by Bochner's theorem that there exists a finite, positive, rotation invariant Radon measure $\mu$ on $R^{N}$ such that

$$
f(x)=\int_{R} e^{i x \cdot t} d \mu(t) \text { for all } x \in G .
$$

One main purpose of this paper is to prove that if $f$ is a continuous radial function on a ball $G=S_{N}(a)=\left\{x \in R^{N}|| x \mid<a\right\}, 0<a \leq \infty$, such that

$$
\int_{G / 2} \int_{G / 2} f(x-y) \phi(x) \overline{\phi(y)} d x d y \geq 0
$$

for all radial functions $\phi \in C_{0}^{\infty}(G / 2)$, then there exist finite, positive, rotation invariant Radon measures $\mu_{1}$ and $\mu_{2}$ on $R^{N}$ such that

$$
f(x)=\int_{R}{ }^{i x \cdot t} d \mu_{1}(t)+\int_{R}{ } e^{x \cdot t} d \mu_{2}(t) \text { for all } x \in G .
$$

The measure $\mu_{2}$ may be taken to be the zero measure if in addition to ( 3 ) the inequality

$$
\int_{G / 2} \int_{G / 2} f(x-y)(-\Delta \phi)(x) \overline{\phi(y)} d x d y \geq 0
$$

holds for all radial functions $\phi \in C_{0}^{\infty}(G / 2)$.

Here $\Delta$ is the Laplace operator in $R^{N}: \Delta \phi=\Sigma \partial^{2} \phi / \partial x_{i}^{2}$.

Condition (5) can be rewritten in the form

$$
\sum_{i=1}^{N} \int_{G / 2} \int_{G / 2} f(x-y) \phi_{x_{i}}(x) \overline{\phi_{x_{i}}(y)} d x d y \geq 0
$$

for all radial functions $\phi \in C_{0}^{\infty}(G / 2)$ : Indeed 


$$
\begin{aligned}
\int_{G / 2} \int_{G / 2} f(x-y)(-\Delta \phi)(x) \overline{\phi(y)} d x d y=\int_{G} f(x)\left((-\Delta \phi) * \phi^{*}\right)(x) d x \\
=\int_{G} f(x) \sum_{i=1}^{N}\left(\phi_{x_{i}} * \phi_{x_{i}}^{*}\right)(x) d x=\sum_{i=1}^{N} \int_{G / 2} \int_{G / 2} f(x-y) \phi_{x_{i}}(x) \overline{\phi_{x_{i}}(y)} d x d y
\end{aligned}
$$

for $\phi \in C_{0}^{\infty}(G / 2)$. Here $\phi^{*}(x)=\overline{\phi(-x)}$ and $*$ denotes convolution.

Since for a continuous radial function $f$ condition (2) clearly implies (3) and (6), our theorem not only yields the theorem of Rudin but actually strongly improves it.

If $N=1$, the first part of our theorem, viz. (3) $\Rightarrow(4)$, is precisely the theorem of Krein [12] on the integral representation of evenly positive definite functions (cf. also [8, p. 196$]$ and [1, Theorem 318, p. 689]).

$\S \S 2-4$ are devoted to preliminaries. The main result on functions positive definite relative to the orthogonal group are proved in $\$ 5$. In $\$ 6$ the results of $\$ 5$ are extended to distributions. In particular Rudin's theorem on the extension of radial positive definite functions is extended to rotation invariant positive definite distributions.

In a sequel to this paper the main results of $\$ 5$ will be further extended.

2. Expansion into generalized eigenvectors. To prove our result we shall use the theory of expansions into generalized eigenvectors first developed in the fundamental paper by Gel'fand and Kostjučenko [7] and expanded later by others, notably Ju. M. Berezanskir. For a detailed account, reference to the literature and a more motivated discussion we refer the reader to the monographs by Berezanskir [1] and Gel'fand and Vilenkin [8].

The theorem we need has been proved in one form or another by various authors. However, we have been unable to find a precise reference for the theorem we need. The theorems in the literature are either different from ours or imprecise or faulty in detail. We shall therefore give a proof of the pertinent theorem.

When referring to nuclear spaces we shall always mean "nuclear in the sense of Grothendieck" [9]. See also Trèves [20, p. 510] or Pietch [16].

If $E$ is a locally convex space we denote by $E^{\prime}$ its topological dual, that is to say the vector space of all continuous linear functionals on $E$. If $x^{\prime} \in E^{\prime}$ we shall denote by $\left\langle x^{\prime}, x\right\rangle$ its value at the point $x$ of $E$. If $A: E \rightarrow F$ is a continuous linear mapping of a locally convex space $E$ into a locally convex space $F$ we denote by ${ }^{t} A$ the transpose of $A$. That is, ${ }^{t} A$ is the linear mapping of $F^{\prime}$ into $E^{\prime}$ defined by

$$
\left\langle{ }^{t} A y^{\prime}, x\right\rangle=\left\langle y^{\prime}, A x\right\rangle \text { for all } x \in E \text { and } y^{\prime} \in F^{\prime} .
$$

An involution in $E$ is a mapping $x \rightarrow x^{*}$ of $E$ into $E$ such that $\left(x^{*}\right)^{*}=x$, $(x+y)^{*}=x^{*}+y^{*}$ and $(\lambda x)^{*}=\bar{\lambda} x^{*}$ for all $x$ and $y$ in $E$ and all complex numbers $\lambda$. 
By a pseudo-inner product we shall mean a positive Hermitian sesquilinear form, i.e. a Hermitian sesquilinear form $f$ on $E$ such that $f(x, x) \geq 0$ for all $x \in E$. We shall write $(x, y)$ for $f(x, y)$.

We assume the reader is familiar with J. von Neumann's theory of a direct integral of Hilbert spaces [14, pp. 404-438]. A short but precise account is given by L. Garding in [6, pp. 1-2].

Theorem 1 (Expansion into generalized eigenvectors). Suppose

1. E is a separable nuclear space,

2. $(x, y)$ is a pseudo-inner product on $E$ which is continuous (in botb variables jointly in the topology of $E \times E$ ), and

3. $A$ is a continuous linear mapping of $E$ into $E$ such that $(A x, y)=(x, A y)$ for all $x$ and $y$ in $E$.

If in addition

4. there exists an involution $x \rightarrow x^{*}$ in $E$ such that $\left(x^{*}, y^{*}\right)=(y, x)$ and $A x^{*}=(A x)^{*}$ for all $x$ and $y$ in $E$, or

$4^{\prime} .(A x, x) \geq 0$ for all $x \in E$,

then there exists a finite positive Radon measure $\mu$ on $R$ and a $\mu$-measurable function $d$ with values in $\{1,2,3, \ldots, \infty\}$, and, for every real number $\lambda$, a sequence $e_{k}^{\prime}(\lambda), k=1,2, \cdots$, of elements in $E^{\prime}$ such that $e_{k}^{\prime}(\lambda)=0$ for $k>d(\lambda)$, and

5. $\lambda \rightarrow\left\langle e_{k}^{\prime}(\lambda), x\right\rangle$ is $\mu$-measurable for every $x \in E$ and every $k=1,2, \ldots$,

6. there exists a $\mu$-null set $N_{0}$ such that for $\lambda \notin N_{0}$, the sequence $e_{k}^{\prime}(\lambda)$, $1 \leq k<d(\lambda)+1$, is linearly independent,

7. ${ }^{t} A e_{k}^{\prime}(\lambda)=\lambda e_{k}^{\prime}(\lambda)$ for all $k=1,2, \ldots$ and $\lambda \in R$,

8. the series $\sum_{k=1}^{d(\lambda)}\left\langle e_{k}^{\prime}(\lambda), x\right\rangle\left\langle\overline{e_{k}^{\prime}(\lambda), y}\right\rangle$ converges absolutely for every $\lambda \in R$ and $x$ and $y$ in $E$,

9. the function $\lambda \rightarrow \Sigma_{k=1}^{d(\lambda)} \mid\left\langle e_{k}^{\prime}(\lambda), x\right\rangle\left\langle\left\langle\overline{e_{k}^{\prime}(\lambda), y}\right\rangle\right|$ belongs to $L^{1}(\mu)$, and

$$
\left.(x, y)=\int_{-\infty}^{\infty} \sum_{k=1}^{d(\lambda)}\left\langle e_{k}^{\prime}(\lambda), x\right\rangle \overline{\left\langle e_{k}^{\prime}(\lambda), y\right.}\right\rangle d \mu(\lambda)
$$

for all $x$ and $y$ in $E$.

If $4^{\prime}$ bolds $\mu$ may be chosen such that the support of $\mu$ is contained in $[0, \infty)$.

Proof. We first assume that the pseudo-inner product $(x, y)$ is nondegenerate, i.e., is an inner product. Let $H$ be the Hilbert space which is the completion of $E$ with respect to the norm defined by the inner product $(x, y)$. We denote the inner product in $H$ again by $(x, y)$. The natural injection $j: x \rightarrow x$ of $E$ into $H$ is continuous since the inner product $(x, y)$ is continuous. It follows that $j$ is 
a nuclear mapping [9, Chapter II, p. 35]. See also [20, Theorem 50.1, p. 511]. Thus there exists a sequence $\left(\lambda_{n}\right)_{n \geq 1}$ of complex numbers such that $\sum_{k=1}^{\infty}\left|\lambda_{n}\right|<\infty$, a bounded sequence $\left(y_{n}\right)_{n \geq 1}$ of elements in $H$ (i.e. $\left\|y_{n}\right\|=\left(y_{n}, y_{n}\right)^{1 / 2} \leq M$ for all $n$ ) and an equicontinuous sequence $\left(x_{n}^{\prime}\right)_{n \geq 1}$ of elements in $E^{\prime}$ (i.e., $\left|\left\langle x_{n}^{\prime}, x\right\rangle\right| \leq p(x)$ for all $n$ and $x \in E$, where $p$ is a continuous seminorm on $E$ ) such that

$$
x=j(x)=\sum_{n=1}^{\infty} \lambda_{n}\left\langle x_{n}^{\prime}, x\right\rangle y_{n} \text { for all } x \in E,
$$

where the series converges in the metric of $H$.

If the condition 4 holds we may extend the involution $x \rightarrow x^{*}$ in $E$ by continuity to an involution in $H$ which satisfies 4 for all $x$ and $y$ in $H$. It follows that $A$-considered as a symmetric operator in $H$ with domain $E$-has a selfadjoint extension [13, p. 41]. Let $T$ be any such selfadjoint extension.

If condition $4^{\prime}$ holds, $A$-considered as a symmetric operator in $H$ with domain $E$-has a positive selfadjoint extension by Friedrich's Theorem [13, p. 35]. In this case we denote by $T$ any positive selfadjoint extension of $A$.

In either case let $\hat{H}=\int_{-\infty}^{\infty} \bigoplus \hat{H}(\lambda) d \mu(\lambda)$ be a direct integral Hilbert space which diagonalizes $T$. (Note that $H$ is a separable Hilbert space because the range of a nuclear mapping is clearly always separable and therefore $j(E)$ is a separable dense subspace of $H$. We have not used the fact that $E$ is separable (in its initial topology) this fact of course also implies that $H$ is separable.) Thus there exists a unitary mapping $U$ of $H$ onto $\hat{H}$ such that $\hat{T}=U T U^{-1}$ is the multiplication operator $[\hat{x}(\lambda)] \rightarrow[\lambda \hat{x}(\lambda)]$. Here $[\hat{x}(\lambda)]$ denotes the equivalence class $\hat{x}$ in $\hat{H}$ which contains the measurable vector field $\lambda \rightarrow \hat{x}(\lambda)$. We also write $\int \bigoplus \hat{x}(\lambda) d \mu(\lambda)$ for $[\hat{x}(\lambda)]$. The domain $D(\hat{T})$ consists of all $\hat{x}=[\hat{x}(\lambda)] \in \hat{H}$ such that $\int_{-\infty}^{\infty} \lambda^{2}\|\hat{x}(\lambda)\|^{2} d \mu(\lambda)<\infty$. $\mu$ is a finite positive Radon measure on $R$ whose support is equal to the spectrum of $T$. Thus, in case condition $4^{\prime}$ is satisfied, the spectrum of $\mu$ is contained in $[0, \infty)$.

If $x \in H$ we denote by $\hat{x}$ the element $U x \in \hat{H}$. If $x \in E$, then

$$
U x=\sum_{n=1}^{\infty} \lambda_{n}\left\langle x_{n}^{\prime}, x\right) ! J y_{n} \text { by (7). }
$$

Thus

$$
\hat{x}=\sum_{n=1}^{\infty} \lambda_{n}\left\langle x_{n}^{\prime}, x\right\rangle \hat{y}_{n} \text { for all } x \in E,
$$

where the series converges in the metric of $\hat{H}$.

Suppose $\hat{y}_{n}=\int \bigoplus \hat{y}_{n}(\lambda) d \mu(\lambda)$ for $n=1,2, \ldots$. Then 


$$
\begin{aligned}
\int_{-\infty}^{\infty} \sum_{n=1}^{\infty}\left|\lambda_{n}\right|\left\|\hat{y}_{n}(\lambda)\right\|^{2} d \mu(\lambda) & =\sum_{n=1}^{\infty}\left|\lambda_{n}\right| \int_{-\infty}^{\infty}\left\|y_{n}(\lambda)\right\|^{2} d \mu(\lambda)=\sum_{n=1}^{\infty}\left|\lambda_{n}\right|\left\|\hat{y}_{n}\right\|^{2} \\
& =\sum_{n=1}^{\infty}\left|\lambda_{n}\right|\left\|y_{n}\right\|^{2} \leq M^{2} \sum_{n=1}^{\infty}\left|\lambda_{n}\right|<\infty .
\end{aligned}
$$

Therefore,

$$
C^{2}(\lambda)=\sum_{n=1}^{\infty}\left|\lambda_{n}\right|\left\|\hat{y}_{n}(\lambda)\right\|^{2}<\infty \quad \mu \text {-a.e. }
$$

Hence, if we modify the vectors $\hat{y}_{1}(\lambda), \hat{y}_{2}(\lambda), \ldots$ at a $\mu$-null set (for example we may set $\hat{y}_{n}(\lambda)=0$ for every $n=1,2, \ldots$ and for $\lambda$ such that $\left.C^{2}(\lambda)=\infty\right)$, we may assume without loss of generality that $C^{2}(\lambda)<\infty$ for all $\lambda \in R, C(\lambda) \geq 0$.

For every real number $\lambda$ and $x \in E$, set

$$
u_{\lambda}(x)=\sum_{n=1}^{\infty} \lambda_{n}\left\langle x_{n}^{\prime}, x\right\rangle \hat{y}_{n}(\lambda) \text {. }
$$

$u_{\lambda}(x) \in \hat{H}(\lambda)$ and its $\hat{H}(\lambda)$-norm

$$
\begin{aligned}
\left\|u_{\lambda}(x)\right\| & \leq \sum_{n=1}^{\infty}\left|\lambda_{n}\right|\left|\left\langle x_{n}^{\prime}, x\right\rangle\right|\left\|\hat{y}_{n}(\lambda)\right\| \leq p(x) \sum_{n=1}^{\infty}\left|\lambda_{n}\right|\left\|\hat{y}_{n}(\lambda)\right\| \\
& \leq p(x)\left(\sum_{n=1}^{\infty}\left|\lambda_{n}\right|\right)^{1 / 2}\left(\sum_{n=1}^{\infty}\left|\lambda_{n}\right|\left\|\hat{y}_{n}(\lambda)\right\|^{2}\right)^{1 / 2} \\
& \leq C(\lambda)\left(\sum_{n=1}^{\infty}\left|\lambda_{n}\right|\right)^{1 / 2} p(x)=M(\lambda)_{p}(x)
\end{aligned}
$$

where $M(\lambda)=C(\lambda)\left(\sum_{n=1}^{\infty}\left|\lambda_{n}\right|\right)^{1 / 2}<\infty$ for every $\lambda \in R$.

Thus $u_{\lambda}: E \rightarrow \hat{H}(\lambda)$ defines a continuous linear mapping of $E$ into $\hat{H}(\lambda)$ for every $\lambda$.

We claim that

$$
\hat{x}=\int \bigoplus u_{\lambda}(x) d \mu(\lambda) \text { for every } x \in E .
$$

Indeed, for a given $x \in E$ suppose $\hat{x}=\int \bigoplus \hat{x}(\lambda) d \mu(\lambda)$.

$$
S_{n}(x)=\sum_{k=1}^{n} \lambda_{k}\left\langle x_{k}^{\prime}, x\right\rangle \hat{y}_{k} \rightarrow \hat{x} \quad \text { in the metric of } \hat{H}
$$

as $n \rightarrow \infty$. It follows (as in the case of an ordinary $L^{2}$-space) that there exists a subsequence $\left(S_{n_{k}}(x)\right)$ of $\left(S_{n}(x)\right)$ such that 


$$
S_{n_{k}}(x)(\lambda)=\sum_{m=1}^{n_{k}} \lambda_{m}\left\langle x_{m}^{\prime}, x\right\rangle \hat{y}_{m}(\lambda) \rightarrow \hat{x}(\lambda) \quad \mu \text {-a.e. }
$$

But $S_{n_{k}}(x)(\lambda) \rightarrow u_{\lambda}(x)$ for every $\lambda \in R$. Therefore $u_{\lambda}(x)=\hat{x}(\lambda) \mu$-a.e. for a fixed $x \in E$. This proves (8).

For every $\lambda \in R$ let $d(\lambda)=\operatorname{dim} \hat{H}(\lambda)$ (Hilbert space dimension). $d$ is $\mu$-measurable-it is "the dimension function of $T$ ". We may assume that, for every $\lambda \in R, \hat{H}(\lambda)$ is the sequence Hilbert space of dimension $d(\lambda)$ consisting of all square summable sequences $c(\lambda)=\left(c(\lambda)_{k}\right)_{k \geq 1}$ with $c(\lambda)_{k}=0$ for $k>d(\lambda)$ and the canonical inner product $(b(\lambda), c(\lambda))=\sum_{k=1}^{\infty} b(\lambda)_{k} \overline{c(\lambda)_{k}}$.

The mapping

$$
c(\lambda) \rightarrow c(\lambda)_{k}
$$

is clearly a continuous linear functional on $\hat{H}(\lambda)$. Therefore the mapping

$$
e_{k}^{\prime}(\lambda): x \rightarrow u_{\lambda}(x)_{k}
$$

as the composite of the two continuous linear mappings $u_{\lambda}$ and (9) is a continuous linear functional on $E$ for every $\lambda \in R$ and every $k=1,2,3, \ldots\left(e_{k}^{\prime}(\lambda)=0\right.$ if $k>d(\lambda))$. Thus $e_{k}^{\prime}(\lambda) \in E^{\prime}$ for all $\lambda \in R$ and $k=1,2,3, \cdots$ and $\lambda \rightarrow\left\langle e_{k}^{\prime}(\lambda), x\right\rangle=$ $u_{\lambda}(x)_{k}$ is $\mu$-measurable for every $x \in E$ and every $k$.

Now, $\left\langle e_{k}^{\prime}(\lambda), A x\right\rangle=u_{\lambda}(A x)_{k}$ and

$$
(A x)=\int \bigoplus \lambda u_{\lambda}(x) d \mu(\lambda) \text { for every } x \in E
$$

Hence $u_{\lambda}(A x)_{k}=\lambda u_{\lambda}(x)_{k}\left(=\lambda\left\langle e_{k}^{\prime}(\lambda), x\right\rangle\right) \mu$-a.e.; i.e. for all $\lambda \notin N_{x, k}$, where $N_{x, k}$ is a $\mu$-null set. Thus, $\left\langle e_{k}^{\prime}(\lambda), A x\right\rangle=\lambda\left\langle e_{k}^{\prime}(\lambda), x\right\rangle$ if $\lambda \notin N_{x, k}, x \in E, k=1,2, \ldots$ Since $E$ is separable there exists a countable dense set $\left\{x_{1}, x_{2}, x_{3}, \ldots\right\}$ in $E$. Let

$$
N^{\prime}=\bigcup_{n, k=1}^{\infty} N_{x_{n, k}}
$$

$N^{\prime}$ is a $\mu$-null set and

$$
\left\langle e_{k}^{\prime}(\lambda), A x_{n}\right\rangle=\lambda\left\langle e_{k}^{\prime}(\lambda), x_{n}\right\rangle
$$

for all $\lambda \notin N^{\prime}$ and every $n=1,2,3, \ldots$ and $k=1,2,3, \ldots$.

Therefore, since the $x_{n}$ 's are dense in $E$, by continuity $\left\langle e_{k}^{\prime}(\lambda), A x\right\rangle=$ $\lambda\left\langle e_{k}^{\prime}(\lambda), x\right\rangle$ for all $\lambda \notin N^{\prime}$, every $k=1,2,3, \cdots$, and all $x \in E$.

We now modify $e_{k}^{\prime}(\lambda)$ for $\lambda \in N^{\prime}$ by setting $e_{k}^{\prime}(\lambda)=0$ for $\lambda \in N^{\prime}$ and every $k=1,2, \ldots$. Then 


$$
\left\langle e_{k}^{\prime}(\lambda), A x\right\rangle=\lambda\left\langle e_{k}^{\prime}(\lambda), x\right\rangle
$$

for all $\lambda \in R$, all $x \in E$, and every $k=1,2,3, \cdots$,

and

$$
\sum_{k=1}^{d(\lambda)} \mid\left\langle e_{k}^{\prime}(\lambda), x\right\rangle\left\langle\overline{e_{k}^{\prime}(\lambda), y}\left|\leq \sum_{k=1}^{d(\lambda)}\right| u_{\lambda}(x)_{k} \overline{u_{\lambda}(y)} \bar{F}_{k}\right| \leq\left\|u_{\lambda}(x)\right\|\left\|u_{\lambda}(y)\right\|
$$

for all $\lambda \in R$ and all $x$ and $y$ in $E$. We have thus proved 5, 7 and 8 .

$$
\int_{-\infty}^{\infty}\left\|u_{\lambda}(x)\right\|\left\|u_{\lambda}(y)\right\| d \mu(\lambda) \leq\left(\int_{-\infty}^{\infty}\left\|u_{\lambda}(x)\right\|^{2} d \mu(\lambda)\right)^{1 / 2}\left(\int_{-\infty}^{\infty}\left\|u_{\lambda}(x)\right\|^{2} d \mu(\lambda)\right)^{1 / 2}=\|\hat{x}\|\|\hat{y}\| \text {. }
$$

Therefore $\lambda \rightarrow \sum_{n=1}^{d(\lambda)}\left|\left\langle e_{k}^{\prime}(\lambda), x\right\rangle \overline{\left\langle e_{k}^{\prime}(\lambda), y\right\rangle}\right|$ belongs to $L^{1}(\mu)$ for every $x$ and $y$ in $E$ and since $\left\langle e_{k}^{\prime}(\lambda), x\right\rangle=u_{\lambda}(x)_{k}$ for all $x \in E, k=1,2,3, \cdots$, and $\lambda \notin N^{\prime}$, $\mu\left(N^{\prime}\right)=0$,

$$
\left.(x, y)=\int_{-\infty}^{\infty}\left(u_{\lambda}(x), u_{\lambda}(y)\right) d \mu(\lambda)=\int_{-\infty}^{\infty} \sum_{k=1}^{d(\lambda)}\left\langle e_{k}^{\prime}(\lambda), x\right\rangle \overline{\left\langle e_{k}^{\prime}(\lambda), y\right.}\right\rangle d \mu(\lambda)
$$

for all $x$ and $y$ in $E$. This establishes the theorem for the case the pseudoinner product is nondegenerate except for 6 .

To prove 6 let $\Lambda_{k}=\{\lambda \in R \mid d(\lambda) \geq k\} . \Lambda_{k}$ is a $\mu$-measurable set. Let $\chi_{k}$ be the characteristic function of $\Lambda_{k}$ (relative to $R$ ) and let $v_{k}(\lambda)$ be the vector in $\hat{H}(\lambda)$ whose $k$ th component is $\chi_{k}(\lambda)$ and all other components are zero. The sequence $v_{k}(\lambda), 1 \leq k<d(\lambda)+1$, is an orthonormal basis in $\hat{H}(\lambda)$ for every $\lambda \in R$.

Let $v_{k}=\int \bigoplus v_{k}(\lambda) d \mu(\lambda)$ for $k=1,2,3, \ldots$. The set $\left\{x_{1}, x_{2}, x_{3}, \ldots\right\}$ is clearly dense in $H$; hence for a fixed $n$ there exists a subsequence $\left(x_{n_{k}}\right)$ of $\left(x_{n}\right)$ which converges to $U^{-1} v_{n}$ in the metric of $H$. Therefore $\hat{x}_{n_{k}}=U x_{n_{k}} \rightarrow v_{n}$ in the metric of $\hat{H}$ as $k \rightarrow \infty$. Since $\hat{x}_{n_{k}}=\int \bigoplus u_{\lambda}\left(x_{n_{k}}\right) d \mu(\lambda)$ there exists (as noted above $)$ a subsequence $\left(u_{\lambda}\left(x_{m_{k}}\right)\right)$ of $\left(u_{\lambda}\left(x_{n_{k}}\right)\right)$ such that $u_{\lambda}\left(x_{m_{k}}\right) \rightarrow v_{n}(\lambda)$ in

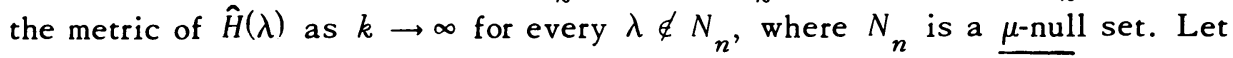
$N^{\prime \prime}=\bigcup_{n=1}^{\infty} N_{n}$, then it follows from what precedes that $v_{n}(\lambda) \in \overline{u_{\lambda}(E)}$ for all $n$ if $\lambda \notin N^{\prime \prime} \overline{\left(u_{\lambda}(E)\right.}$ denotes the closure of $u_{\lambda}(E)$ in $\left.H(\lambda)\right)$. Thus $u_{\lambda}(E) \supset \hat{H}(\lambda)$ and therefore $u_{\lambda}(E)=\hat{H}(\lambda)$ for $\lambda \notin N^{\prime \prime}$.

Let $N_{0}=N^{\prime} \cup N^{\prime \prime} . N_{0}$ is a $\mu$-null set and the elements $e_{k}^{\prime}(\lambda), 1 \leq k<d(\lambda)$ +1 , are linearly independent if $\lambda \notin N_{0}$. Otherwise there exists for some $\lambda \notin N_{0}$ a sequence $\left(c_{k}\right)_{k \geq 1}$ of complex numbers which are not all zero and such that $c_{k}=0$ for $k>n$, where $n$ is an integer $\leq d(\lambda)$ and $\Sigma_{k \geq 1} c_{k} e_{k}^{\prime}(\lambda)=0$. Therefore

$$
\sum_{k \geq 1} c_{k}\left\langle e_{k}^{\prime}(\lambda), x\right\rangle=\sum_{k \geq 1} c_{k} u_{\lambda}(x)=0 \text { for all } x \in E \text {. }
$$

Thus $c=\left(\bar{c}_{k}\right)_{k \geq 1} \in \hat{H}(\lambda), c \neq 0$ and $c$ is orthogonal to $u_{\lambda}(E)$. This contradicts $\overline{u_{\lambda}(E)}=\hat{H}(\lambda)$. 
Assume now that the pseudo-inner product is degenerate. Let $N=$ $\{x \in E \mid(x, x)=0\} . N$ is a closed linear subspace of $E$ and therefore the quotient space $\dot{E}=E / N$ is nuclear [9, Chapter II, p. 47] (see also [20, Proposition 50.4, p. 514$]$ or [16, Satz 5.1.3, p. 77]). $\dot{E}$ is separable because $E$ is separable. $A N \subset N$ for if $x \in N$ then $(A x, A x)=\left(A^{2} x, x\right) \leq\left(A^{2} x, A^{2} x\right)^{1 / 2}(x, x)^{1 / 2}=0$.

Let $\dot{A}: \dot{E} \rightarrow \dot{E}$ be the quotient mapping of $A \bmod N: \dot{A} \dot{x}=\dot{A x}$ where $\dot{x}=$ $x+N$ is the coset in $\dot{E}$ which contains $x . \dot{A}$ is a continuous linear mapping of $\dot{E}$ into $\dot{E}$.

On $\dot{E}$ define an inner product by setting $(\dot{x}, \dot{y})=(x, y)$. Clearly $(\dot{x}, \dot{y})$ is continuous in $\dot{x}$ and $\dot{y}$, and if condition $4^{\prime}$ holds for $A$ it also holds for $\dot{A}$ because $(\dot{A} \dot{x}, \dot{x})=(A x, x)$ for all $\dot{x} \in \dot{E}$. If condition 4 holds define an involution in

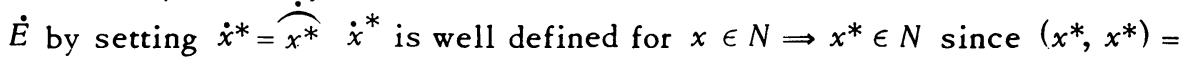
$(x, x)$. Moreover,

$$
\left(\dot{x}^{*}, \dot{y}^{*}\right)=\left(x^{*}, y^{*}\right)=(y, x)=(\dot{y}, \dot{x})
$$

and

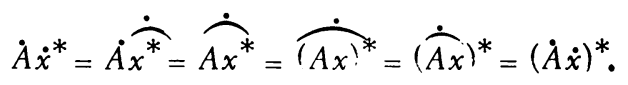

Thus condition 4 holds for $\dot{A}$.

It follows from what precedes that the conclusions of the theorem are valid for $E$ and $A$, etc., replaced by $\dot{E}$ and $\dot{A}$, etc., respectively. Thus there exists a finite, positive Radon measure $\mu$ on $R$ whose support is contained in $[0, \infty)$ if $4^{\prime}$ holds (for $A$ ), a $\mu$-measurable function $d$ with values in $\{1,2,3, \ldots, \infty\}$, and for every $\lambda \in R$, a sequence $\dot{e}_{k}^{\prime}(\lambda), k=1,2,3, \ldots$, in $\dot{E}^{\prime}$ such that $\dot{e}_{k}^{\prime}(\lambda)=0$ for $k>d(\lambda)$ and conditions 5-9 hold.

Let $e_{k}^{\prime}(\lambda) \in E^{\prime}$ be the linear functional on $E$ defined by

$$
\left\langle e_{k}^{\prime}(\lambda), x\right\rangle=\left\langle\dot{e}_{k}^{\prime}(\lambda), \dot{x}\right\rangle \text { for every } x \in E
$$

then $e_{k}^{\prime}(\lambda)=0$ for $k>d(\lambda)$.

5. $\lambda \rightarrow\left\langle\dot{e}_{k}^{\prime}(\lambda), x\right\rangle=\left\langle\dot{e}_{k}^{\prime}(\lambda), \dot{x}\right\rangle$ is $\mu$-measurable for every $k=1,2, \ldots$

6. $e_{k}^{\prime}(\lambda), 1 \leq k<d(\lambda)+1$, are linearly independent for $\lambda \notin N_{0}$ (because $e_{k}^{\prime}(\lambda), 1 \leq k<d(\lambda)+1$, are linearly dependent if and only if $\dot{e}_{k}^{\prime}(\lambda), 1 \leq k<$ $d(\lambda)+1$, are linearly dependent).

7. ${ }^{t} A e_{k}^{\prime}(\lambda)=\lambda e_{k}^{\prime}$ for all $\lambda \in R$ and all $k$, because $\left\langle e_{k}^{\prime}(\lambda), A x\right\rangle=$ $\left\langle\dot{e}_{k}^{\prime}(\lambda), \dot{A} \dot{x}\right\rangle=\lambda\left\langle\dot{e}_{k}^{\prime}(\lambda), \dot{x}\right\rangle=\lambda\left\langle e_{k}^{\prime}(\lambda), x\right\rangle$ for all $x \in E, \lambda \in R$ and $k=1,2, \ldots$

Conditions 8 and 9 follow from the fact that

$$
\sum_{k=1}^{d(\lambda)}\left|\left\langle e_{k}^{\prime}(\lambda), x\right\rangle \overline{\left.e_{k}^{\prime}(\lambda), y\right\rangle}\right|=\sum_{k=1}^{d(\lambda)}\left|\left\langle\dot{e}_{k}^{\prime}(\lambda), \dot{x}\right\rangle \overline{\left\langle\dot{e}_{k}^{\prime}(\lambda), \dot{y}\right\rangle}\right| .
$$

This completes the proof. 
3. Rotation invariant distributions. Let $O(N)$ be the orthogonal group operating in $R^{N}, f$ a function defined on the ball $S_{N}(a)$. For every $\left.\rho \in O^{\prime} N\right)$ we define the function $\rho f$ by $\rho f(x)=f\left(\rho^{-1} x\right)$ for $x \in S_{N}(a)$. $f$ is said to be rotation invariant or radial if $\rho f=f$ for all $\rho \in O(N)$. In that case $f(x)=F(|x|)$ for all $x \in S_{N}(a)$, where $F(r)$ is a function on $0 \leq r<a$. In fact, $F(r)=f(r, 0, \ldots, 0)$.

Let $d \rho$ be the Haar measure on $O(N)$ normalized such that $\int_{O(N)} d \rho=1$; we recall that $O(N)$ is compact. If $f$ is a continuous function on $S_{N}(a)$ we set for every $x \in S_{N}(a)$

$$
f^{\#}(x)=\int_{O(N)} \rho f(x) d \rho .
$$

Clearly $f^{\#}$ is a continuous rotation invariant function on $S_{N}(a)$ which is a $C^{\infty}$. function if $f$ is a $C^{\infty}$-function.

Let $\mathscr{D}^{\sharp}\left(S_{N}(a)\right)$ be the space of rotation invariant complex valued $C^{\infty}$-functions on $S_{N}(a)$ with compact support. $\mathscr{T}^{\#}\left(S_{N}(a)\right)$ is a vector subspace of $\mathscr{D}\left(S_{N}(a)\right)$ (the usual space of test functions on $S_{N}(a)$ of distribution theory with the Schwartz topology). We endow $\mathscr{D}^{\sharp}\left(S_{N}(a)\right)$ with the topology induced by $\mathcal{D}\left(s_{N}(a)\right)$.

If a sequence $\left(\phi_{n}\right)_{n \geq 1}$ in $\mathcal{D}\left(S_{N}(a)\right)$ converges to 0 in $\mathscr{D}\left(S_{N}(a)\right)$, i.e. $\phi_{n} \rightarrow 0$ uniformly, as well as all its derivatives and keeps its support in a fixed compact set, the same is true for the sequence $\left(\phi_{n}^{\#}\right)_{n \geq 1}$. That is, the mapping \#: $\phi \rightarrow \phi^{\#}$ is a linear sequentially continuous mapping of $\mathscr{D}\left(s_{N}(a)\right)$ onto $\mathfrak{D}^{\sharp}\left(s_{N}(a)\right)$, whencesince $\mathscr{D}\left(S_{N}(a)\right)$ is bornological-\# is a continuous projection of $\mathscr{D}\left(S_{N}(a)\right)$ onto $\mathcal{D}^{\#}\left(S_{N}(a)\right)$. Clearly $(\rho \phi)^{\#}=\phi^{\#}$ for all $\phi \in \mathcal{D}\left(S_{N}(a)\right)$ and $\rho \in O(N)$ since the Haar measure $d \rho$ is unimodular.

If $T$ is a distribution in $S_{N}(a)$, i.e. $T \in \mathscr{D}^{\prime}\left(S_{N}(a)\right)$, and $\left.\rho \in O^{\prime} N\right)$ we define a new distribution $\rho T$ on $S_{N}(a)$ by $\phi \rightarrow\left\langle T, \rho^{-1} \phi\right\rangle$ for all $\phi \in \mathscr{D}\left(S_{N}(a)\right)$. We note that if $T$ corresponds to a locally integrable function $f$ on $S_{N}(a), \rho T$ corresponds to $\rho f . T$ is said to be rotation invariant if $\rho T=T$ for all $\rho \in O(N)$. The rotation invariant distributions on $S_{N}(a)$ form a closed vector subspace $\mathscr{D}^{\prime \#}\left(S_{N}(a)\right)$ of $\mathscr{D}^{\prime}\left(S_{N}(a)\right)\left(\mathfrak{D}^{\prime}\left(S_{N}(a)\right)\right.$ is endowed with the strong dual topology).

Let $\mathfrak{D}^{\#}\left(S_{N}(a)\right)^{\prime}$ be the strong dual of $\mathscr{D}^{\#}\left(S_{N}(a)\right)$ and ${ }^{t} \#: \mathscr{D}^{\#}\left(S_{N}(a)\right)^{\prime} \rightarrow$ $\mathfrak{D}^{\prime}\left(S_{N}(a)\right)$ the transpose of \#.

Proposition 1. ${ }^{t} \#$ is a topological isomorphism of $\mathfrak{D}^{\#}\left(S_{N}(a)\right)^{\prime}$ onto $\mathfrak{L}^{\prime \#}\left(S_{N}(a)\right)$; its inverse is the mapping which associates with every rotation invariant distribution $T$ in $S_{N}(a)$ its restriction to $\mathfrak{D}^{\#}\left(S_{N}(a)\right)$. Thus every element in $\mathscr{D}^{\#}\left(S_{N}(a)\right)^{\prime}$ is the restriction to $\mathscr{D}^{\#}\left(S_{N}(a)\right)$ of a unique element in $\mathfrak{D}^{\prime \sharp}\left(S_{N}(a)\right)$.

Proof. \#: $\mathfrak{D}\left(S_{N}(a)\right) \rightarrow \mathscr{D}^{\#}\left(S_{N}(a)\right)$ is continuous and its range is $\mathscr{D}^{\#}\left(S_{N}(a)\right)$. Therefore ${ }^{t} \#$ is continuous and $1-1$. 
Suppose $T={ }^{t} \#(S), S \in \mathscr{D}^{\sharp}\left(S_{N}(a)\right)^{\prime}$. Then for every $\phi \in \mathscr{D}\left(S_{N}(a)\right)$, and $\rho \in O(N)$,

$$
\langle\rho T, \phi\rangle=\left\langle T, \rho^{-1} \phi\right\rangle=\left\langle S,\left(\rho^{-1} \phi\right)^{\#}\right\rangle=\left\langle S, \phi^{\#}\right\rangle=\langle T, \phi\rangle,
$$

whence $T \in \mathscr{D}^{\prime \#}\left(S_{N}(a)\right)$. Thus the range of ${ }^{t} \#$ is contained in $\mathscr{D}^{\prime \#}\left(S_{N}(a)\right)$.

Suppose $T \in \mathscr{D}^{\prime \#}\left(S_{N}(a)\right)$. Let $S$ be its restriction to $\mathscr{D}^{\sharp}\left(S_{N}(a)\right)$. Then $\langle T, \phi\rangle=\langle T, \rho \phi\rangle$ for every $\phi \in \mathscr{D}\left(S_{N}(a)\right)$ and $\rho \in O(N)$. Therefore

$$
\langle T, \phi\rangle=\int_{O(N)}\langle T, \phi\rangle d \rho=\int_{O(N)}\langle T, \rho \phi\rangle d \rho=\left\langle T, \int_{O(N)} \rho \phi d \rho\right\rangle \quad\left(=\left\langle T, \phi^{\sharp}\right\rangle\right)
$$

for every $\phi \in \mathscr{D}\left(S_{N}(a)\right)$. The last equality holds if the distribution $T$ is a function by Fubini's theorem. The general case is obtained by regularization: Choose a sequence $\left(u_{k}\right)_{k \geq 1}$ in $\mathscr{D}^{\sharp}\left(S_{N}(a)\right)$ such that $u_{k} \rightarrow T$ in $\mathscr{D}^{\prime}\left(S_{N}(a)\right)$; then

$$
\langle T, \phi\rangle=\lim _{k \rightarrow \infty}\left\langle u_{k}, \phi\right\rangle=\lim _{k \rightarrow \infty}\left\langle u_{k}, \phi^{\#}\right\rangle=\left\langle T, \phi^{\#}\right\rangle
$$

for all $\phi \in \mathscr{D}\left(S_{N}(a)\right)$. Thus $\langle T, \phi\rangle=\left\langle T, \phi^{\#}\right\rangle=\left\langle S, \phi^{\#}\right\rangle=\left\langle{ }^{t} \#(S), \phi\right\rangle$ for all $\phi \epsilon$ $\mathscr{D}\left(S_{N}(a)\right)$, whence $T={ }^{t} \#(S)$.

Finally, if $j: \mathscr{D}^{\sharp}\left(S_{N}(a)\right) \rightarrow \mathscr{D}\left(S_{N}(a)\right)$ is the natural injection of $\mathfrak{D}^{\#}\left(S_{N}(a)\right)$ into $\mathscr{D}\left(S_{N}(a)\right)$, the transpose ${ }^{t} j: \mathscr{D}^{\prime}\left(S_{N}(a)\right) \rightarrow \mathscr{D}^{\sharp}\left(S_{N}(a)\right)^{\prime}$ is continuous. If $T \in$ $\mathscr{D}^{\prime \#}\left(S_{N}(a)\right)$, then $\left\langle{ }^{t} j(T), \phi\right\rangle=\langle T, \phi\rangle$ for all $\phi \in \mathfrak{D}^{\sharp}\left(S_{N}(a)\right)$. Thus the restriction of ${ }^{t} j$ to $\mathfrak{D}^{\prime \#}\left(S_{N}(a)\right)$ is precisely the inverse of ${ }^{t} \#$ (restricted to $\left.\mathscr{D}^{\prime \#}\left(S_{N}(a)\right)\right)$. This completes the proof.

4. Further preliminaries. If a function $f \in L^{1}\left(R^{N}\right)$ is radial, $f(x)=F(|x|)$, then its Fourier transform $\hat{f}(t)=\int_{R} N e^{-i t \cdot x} f(x) d x$ is equal to [2, p. 187]

$$
\hat{f}(t)=(2 \pi)^{N / 2} \int_{0}^{\infty} \Omega_{N}(|t| r) F(r) d \mu_{N}(r)=\int_{R}{ } \Omega_{N}(|t||x|) f(x) d x .
$$

Here $d \mu_{N}(r)=\left[2^{(N-2) / 2} \Gamma(N / 2)\right]^{-1} r^{N-1} d r$ and

$$
\begin{aligned}
\Omega_{N}(z)= & \Gamma\left(\frac{N}{2}\right)\left(\frac{2}{z}\right)^{(N-2) / 2} J_{(N-2) / 2}(z) \quad \text { if } z \neq 0 \text { and } \\
& =1 \text { if } z=0, \\
& =1-\frac{z^{2}}{2 N}+\frac{z^{4}}{2 \cdot 4 N(N+2)}-\frac{z^{6}}{2 \cdot 4 \cdot 6 N(N+2)(N+4)}+\cdots
\end{aligned}
$$

$J_{\nu}(z)$ is the Bessel function of index $\nu$.

(The function $\widetilde{F}(\lambda)=\int_{0}^{\infty} \Omega_{N}(\lambda r) F(r) d \mu_{N}(r), 0 \leq \lambda<\infty$, is called the Hankel transform of $F$ of order $(N-2) / 2$. If $F$ has compact support $\widetilde{F}(\lambda)$ exists for all complex numbers $\lambda$; in this case $\widetilde{F}$ is an even entire analytic function.)

5. Functions positive definite relative to the orthogonal group.

Definition 1. A continuous complex valued function $f$ (see the note following 
the proof of Theorem 2) on a ball $S_{N}(a), 0<a \leq \infty$, in $R^{N}$ is said to be positive definite relative to the orthogonal group $(O(N))$ if it is radial and if

$$
\int_{S_{N}(a / 2)} \int s_{N^{(a / 2)}} f(x-y) \phi(x) \overline{\phi(y)} d x d y \geq 0
$$

for all radial $\phi \in C_{0}^{\infty}\left(S_{N}(a / 2)\right)$.

Theorem 2. A continuous function $f$ on a ball $S_{N}(a), 0<a \leq \infty$, in $R^{N}$ is positive definite relative to the orthogonal group if and only if there exists a positive bounded Radon measure $\gamma$ on $R$ such that

$$
f(x)=\int_{-\infty}^{\infty} \Omega_{N}\left(|x| \lambda^{1 / 2}\right) d y(\lambda) \quad \text { for all } x \in S_{N}(a) .
$$

The measure $\gamma$ may be chosen such that its support is contained in $[0, \infty)$ if in addition to (10)

$$
\int_{S_{N}(a / 2)} \int_{S_{N}(a / 2)} f(x-y)(-\Delta \phi)(x) \overline{\phi(y)} d x d y \geq 0
$$

for all radial $\phi \in C_{0}^{\infty}\left(S_{N}(a / 2)\right)$.

Proof. Let $E=\mathscr{D}^{\#}(G)$ where $G=S_{N}(a / 2)$. $E$ is a subspace of $\mathscr{D}(G)$ which is nuclear [9, Chapter II, p. 55] (see also [20, p. 530]), hence $E$ is nuclear. Clearly $E$ is separable. On $E$ we define a pseudo-inner product by setting

$$
(\phi, \psi)=\int_{G} \int_{G} f(x-y) \phi(x) \overline{\psi(y)} d x d y=\int_{S_{N}(a)} f(x) \phi * \psi^{*}(x) d x
$$

for $\phi$ and $\psi$ in $\mathfrak{I}^{\#}(G)$. Here $\psi^{*}(x)=\overline{\psi(-x)}=\overline{\psi(x)}$.

Let $A$ be the linear operator $-\Delta$ in $E$. Here $\Delta$ denotes the Laplacian in $R^{N} . A$ is continuous in $E$ and $(A \phi, \psi)=(\phi, A \psi)$ for all $\phi$ and $\psi$ in $E$ because $\phi_{x_{i}} * \psi=-\phi * \psi_{x_{i}}, \phi_{x_{i}}^{*}=-\left(\phi_{x_{i}}\right)^{*}, i=1, \ldots, N$, and therefore $\Delta \phi *$ $\psi^{*}=\phi * \Delta \psi^{*}=\phi *(\Delta \psi)^{*}$ for all $\phi$ and $\psi$ in $C_{0}^{\infty}\left(R^{N}\right)$. If we introduce in $E$ the involution $\phi \rightarrow \phi^{*}$ then all the conditions 1-4 of Theorem 1 are satisfied. Let $\mu, d, e_{k}^{\prime}(\lambda)$ and $N_{0}$ be as in Theorem 1. The measure $\mu$ has its support in $[0, \infty)$ if $(12)$ holds. $e_{k}^{\prime}(\lambda) \in E^{\prime}=\mathfrak{D}^{\sharp}(G)^{\prime}$ for all $\lambda$ and all $k$. By Proposition 1 there exists for every $\lambda$ and $k$ a unique rotation invariant distribution $e_{k}(\lambda)$ in $G$ whose restriction to $\mathfrak{D}^{\sharp}(G)$ is $e_{k}^{\prime}(\lambda)$. A simple calculation shows that the Laplacian commutes with every rotation: $\Delta(\rho \phi)=\rho(\Delta \phi)$ for all $\phi \in C_{0}^{\infty}\left(R^{N}\right)$ and $\rho \in O(N)$ and hence $(\Delta \phi)^{\ddot{\#}}=\Delta \phi^{\#}$ for every $\phi \in \mathscr{D}(G)$. Hence for every $\phi \in \mathscr{D}(G)$,

$$
\left\langle e_{k}(\lambda),-\Delta \phi\right\rangle=\left\langle e_{k}^{\prime}(\lambda),(-\Delta \phi)^{\#}\right\rangle=\left\langle e_{k}^{\prime}(\lambda), A \phi^{\#}\right\rangle
$$

by Proposition 1. Similarly $\left\langle e_{k}(\lambda), \phi\right\rangle=\left\langle e_{k}^{\prime}(\lambda), \phi^{\#}\right\rangle$ for $\phi \in \mathscr{D}(G)$. But $\left\langle e_{k}^{\prime}(\lambda), A \phi^{\#}\right\rangle=\lambda\left\langle e_{k}(\lambda), \phi^{\#}\right\rangle$ for all $\phi^{\#} \in \mathscr{D}^{\sharp}(G)=E$ by 7 of Theorem 1 . Hence, $\left\langle e_{k}(\lambda),-\Delta \phi\right\rangle=\lambda\left\langle e_{k}(\lambda), \phi\right\rangle$ for all $\phi \in \mathscr{D}(G)$ and all $\lambda \in R$ and $k=1,2, \ldots$ That is-since the transpose of the differential operator $\Delta$ is $\Delta$ - 


$$
(\Delta+\lambda) e_{k}(\lambda)=0 \quad \text { for all } \lambda \in R \text {, and } k=1,2, \ldots,
$$

in the sense of distributions. It follows since $\Delta+\lambda$ is an elliptic differential operator that $e_{k}(\lambda)$ is a $C^{\infty}$-function $e_{k}(\lambda, x)$ in $G$ which satisfies the differential equation (13) in the classical sense. In fact $e_{k}(\lambda, x)$ is an analytic function of $x$ since the coefficients of $\Delta+\lambda$ are analytic [10, Theorem 7.5.1, p. 178].

Since $e_{k}(\lambda, x)=e_{k}\left(\lambda ; x_{1}, x_{2}, \ldots, x_{N}\right)$ is radial,

$$
e_{k}(\lambda, x)=u_{k}(\lambda, r), \quad r=|x|<a / 2,
$$

where $u_{k}(\lambda, r)=e_{k}(\lambda ; r, 0, \ldots, 0)$ is a $C^{\infty}$-function on $0 \leq r<a / 2$ which satisfies the equation

$$
(\Delta+\lambda) e_{k}(\lambda, x)=\frac{d^{2} u_{k}(\lambda, r)}{d r^{2}}+\frac{N-1}{r} \frac{d u_{k}(\lambda, r)}{d r}+\lambda u_{k}(\lambda, r)=0
$$

for $0<r<a / 2$.

If $\lambda=0$, the general solution of (14) is

$$
\begin{array}{ll}
e_{k}(0, x)=u_{k}(0, r)=A_{0}+B / r^{N-2} & \text { if } N \neq 2, \text { and } \\
e_{k}(0, x)=u_{k}(0, r)=A_{0}+B \log r & \text { if } N=2,
\end{array}
$$

for $0<r=|x|<a / 2$. ( $A_{0}$ and $B$ are constants.) It follows since $e_{k}(\lambda, x)$ is a $C^{\infty}$-function in $G$ that $e_{k}(0, x)=A_{0}$ for every $N=1,2,3, \ldots$

If $\lambda \neq 0$ the general solution of (14) is $e_{k}(\lambda, x)=u_{k}(\lambda, r)=r^{-(N-2) / 2} w\left(\lambda^{1 / 2} r\right)$, where $w(r)$ is the general solution of the Bessel equation

$$
r^{2} w^{\prime \prime}(r)+r w^{\prime}(r)+\left(r^{2}-((N-1) / 2)^{2}\right) w(r)=0
$$

(the simple computation is left to the reader). It follows that if $\lambda \neq 0$,

$$
e_{k}(\lambda, x)=u_{k}(\lambda, r)=C_{1} r^{-(N-2) / 2} J_{(N-2) / 2}\left(\lambda^{1 / 2} r\right)+C_{2} r^{-(N-2) / 2} N(N-2) / 2\left(\lambda^{1 / 2} r\right)
$$

for $0<r=|x|<a / 2$. Here $J_{\nu}$ is Bessel's function of index $\nu$ and $N_{\nu}$ is Neumann's function (or Bessel's function of the second kind) of index $\nu . C_{1}$ and $\mathrm{C}_{2}$ are constants.

If $m=(N-2) / 2$ is not an integer,

$$
N_{m}(r)=\frac{\cos m \pi J_{m}(r)-J_{-m}(r)}{\sin m \pi}
$$

and therefore (15) becomes

$$
\begin{aligned}
e_{k}(\lambda, x) & =u_{k}(\lambda, r)=C_{1}^{1} r^{-m} J_{m}\left(\lambda^{1 / 2} r\right)+C_{2}^{1} r^{-m} J_{-m}\left(\lambda^{1 / 2} r\right) \\
& =A_{1} \Omega_{N}\left(\lambda^{1 / 2} r\right)+C_{2}^{1} r^{-m} J_{-m}\left(\lambda^{1 / 2} r\right)
\end{aligned}
$$


for $0<r=|x|<a / 2$. Here $C_{1}^{1}, C_{2}^{1}$ and $A_{1}$ are constants. Now $e_{k}(\lambda, x)$ is a $C^{\infty}$. function in $S_{N}(a)$ and so is $\Omega_{N}\left(\lambda^{1 / 2}|x|\right)$ (for every $N$ ). Therefore $C_{2}^{1} r^{-m} J_{-m}\left(\lambda^{1 / 2} r\right)$ may be extended to $r=0$ so as to be infinitely differentiable at $x=0$. But $\left|r^{-m} J_{-m}\left(\lambda^{1 / 2} r\right)\right| \rightarrow \infty$ as $r \rightarrow 0$ if $m>0$ (i.e. $N \geq 3$ ); and if $N=1$, $r^{-m} J_{-m}\left(\lambda^{1 / 2} r\right)=\left(2 / \pi(\lambda)^{1 / 2}\right)^{1 / 2} \sin \left(\lambda^{1 / 2}|x|\right)$ which is not differentiable at $x=0$. Therefore $C_{2}^{1}=0$ in all cases. Thus

$$
e_{k}(\lambda, x)=A_{1} \Omega_{N}\left(\lambda^{1 / 2}|x|\right) \text { for }|x|<a / 2
$$

If $m=(N-2) / 2$ is an integer then $\left|r^{-m} N_{m}\left(\lambda^{1 / 2} r\right)\right| \rightarrow \infty$ as $r \rightarrow 0$. See [21]. It follows for the same reason as above that $C_{2}$ in (15) must be zero. Thus

$$
e_{k}(\lambda, x)=C_{1} r^{-m} J_{m}\left(\lambda^{1 / 2} r\right)=A_{2} \Omega_{N}\left(\lambda^{1 / 2} r\right),
$$

$A_{2}$ constant, for $r=|x|<a / 2$. Since $\Omega_{N}(0|x|)=1$ we have

$$
e_{k}(\lambda, x)=A(\lambda) \Omega_{N}\left(\lambda^{1 / 2}|x|\right) \text { for }|x|<a / 2 \text { and all } \lambda \in R,
$$

where $A(\lambda)$ is a constant depending upon $\lambda$. Therefore $d(\lambda)=1 \mu$-a.e. and

$$
\left\langle e_{1}(\lambda), \phi\right\rangle=A(\lambda) \int_{S_{N}(a / 2)} \Omega_{N}\left(\lambda^{1 / 2}|x|\right) \phi(x) d x .
$$

By 9 of Theorem 1,

$$
\left\langle e_{1}(\lambda), \phi\right\rangle\left\langle\overline{\left.e_{1}(\lambda), \psi\right\rangle}=|A(\lambda)|^{2} \int_{G} \int_{G} \Omega_{N}\left(|x| \lambda^{1 / 2}\right) \Omega_{N}\left(|y| \lambda^{1 / 2}\right) \phi(x) \bar{\psi}(y) d x d y\right.
$$

belongs to $L^{1}(\mu)$ for all $\phi$ and $\psi$ in $\mathfrak{D}^{\#}(G)$. Hence $\lambda \rightarrow|A(\lambda)|^{2}$ is locally $\mu$ integrable. Let $\gamma$ be the positive Radon measure $d \gamma(\lambda)=|A(\lambda)|^{2} d \mu(\lambda)$ on $R$; then by 9 of Theorem 1 ,

$$
\begin{aligned}
(\phi, \psi) & =\int_{G} \int_{G} f(x-y) \phi(x) \overline{\psi(y)} d x d y \\
& =\int_{-\infty}^{\infty} \int_{G} \int_{G} \Omega_{N}\left(|x| \lambda^{1 / 2}\right) \Omega_{N}\left(|y| \lambda^{1 / 2}\right) \phi(x) \bar{\psi}(y) d x d y d y(\lambda)
\end{aligned}
$$

for all $\phi$ and $\psi$ in $\mathfrak{T}^{\#}(G)$.

Let $x_{0}$ be an arbitrary but fixed point in $G$, and let, for every positive integer $n, \phi_{n}$ be a function in $\mathfrak{T}^{\#}(G)$ such that $\phi_{n} \geq 0$, supp $\phi \subset\left\{x|||x|-\left|x_{0}\right| \mid<1 / n\right\}$ and $\int \phi_{n} d x=1$, then by standard arguments

$$
\int_{G} \Omega_{N}\left(|x| \lambda^{1 / 2}\right) \phi_{n}(x) d x \rightarrow \Omega_{N}\left(\left|x_{0}\right| \lambda^{1 / 2}\right) \quad \text { as } n \rightarrow \infty,
$$

and therefore if we substitute in (16) $\phi_{n}$ for $\phi$ and $\psi$ we obtain by Fatou's lemma

$$
\begin{aligned}
\int_{-\infty}^{\infty}\left(\Omega_{N}\left(\left|x_{0}\right| \lambda^{1 / 2}\right)\right)^{2} d y(\lambda) \leq \lim _{n \rightarrow \infty} \int_{G} \int_{G} f(x-y) \phi_{n}(x) \overline{\phi_{n}(y)} d x d y \\
\quad=\frac{\lim }{n \rightarrow \infty} \int s_{N}(a) \\
\quad \leq \sup _{|x| \leq 2\left|x_{0}\right|}|f(x)|=M\left(x_{0}\right)<\infty .
\end{aligned}
$$


Therefore if $x$ and $y$ are arbitrary points in $G$ then

$$
\begin{aligned}
\int_{-\infty}^{\infty}\left|\Omega_{N}\left(|x| \lambda^{1 / 2}\right) \Omega_{N}\left(|y| \lambda^{1 / 2}\right)\right| d \gamma(\lambda) & \leq\left(\int_{-\infty}^{\infty}\left(\Omega_{N}\left(|x| \lambda^{1 / 2}\right)\right)^{2} d \gamma(\lambda)\right)^{1 / 2}\left(\int_{-\infty}^{\infty}\left(\Omega_{N}\left(|y| \lambda^{1 / 2}\right)\right)^{2} d y(\lambda)\right)^{1 / 2} \\
& \leq M(x)^{1 / 2} M(y)^{1 / 2}<\infty .
\end{aligned}
$$

In particular, $\int_{-\infty}^{\infty} d \gamma(\lambda) \leq M(0)=|f(0)|$; i.e. $\gamma$ is a finite measure.

Now

and therefore

$$
\begin{aligned}
\int_{G} \int_{G}\left|\Omega_{N}\left(|x| \lambda^{1 / 2}\right) \Omega_{N}\left(|y| \lambda^{1 / 2}\right) \phi(x) \overline{\psi(y)}\right| d x d y \\
\leq\left(\int_{G}\left|\Omega_{N}\left(|x| \lambda^{1 / 2}\right) \phi(x)\right|^{2} d x\right)^{1 / 2}\left(\int_{G}\left|\Omega_{N}\left(|y| \lambda^{1 / 2}\right) \psi(y)\right|^{2} d y\right)^{1 / 2}
\end{aligned}
$$

$$
\begin{aligned}
& \int_{-\infty}^{\infty} \int_{G} \int_{G}\left|\Omega_{N}\left(|x| \lambda^{1 / 2}\right) \Omega_{N}\left(|y| \lambda^{1 / 2}\right) \phi(x) \overline{\psi(y)}\right| d x d y d y(\lambda) \\
& \leq\left(\int_{-\infty}^{\infty} \int_{G}\left|\Omega_{N}\left(|x| \lambda^{1 / 2}\right) \phi(x)\right|^{2} d x d y(\lambda)\right)^{1 / 2}\left(\int_{-\infty}^{\infty} \int_{G}\left|\Omega_{N}\left(|y| \lambda^{1 / 2}\right) \psi(y)\right|^{2} d y d y(\lambda)\right)^{1 / 2} \\
& \quad=(\phi, \phi)(\psi, \psi)<\infty \text { for all } \phi \text { and } \psi \text { in } \mathfrak{D}^{H}(G) .
\end{aligned}
$$

Hence from (16) by Fubini's theorem

$$
\int_{G} \int_{G} f(x-y) \phi(x) \overline{\psi(y)} d x d y=\int_{G} \int_{G} K(x, y) \phi(x) \overline{\psi(y)} d x d y
$$

for all $\phi$ and $\psi$ in $\mathfrak{D}^{\sharp}(G)$. Here $K(x, y)=\int_{-\infty}^{\infty} \Omega_{N}\left(|x| \lambda^{1 / 2}\right) \Omega_{N}\left(|y| \lambda^{1 / 2}\right) d y(\lambda)$ for $x$ and $y$ in $G$.

$K(x, y)$ is continuous on $G \times G$, for $(x, y) \rightarrow \int_{0}^{\infty} \Omega_{N}\left(|x| \lambda^{1 / 2}\right) \Omega_{N}\left(|y| \lambda^{1 / 2}\right) d y(\lambda)$ is continuous on $R^{N} \times R^{N}$ by the Lebesgue bounded convergence theorem, since $\left|\Omega_{N}(r)\right| \leq 1$ for all $0 \leq r<\infty$ (cf. (22) below), and

$$
(x, y) \rightarrow \int_{-\infty}^{0} \Omega_{N}\left(|x| \lambda^{1 / 2}\right) \Omega_{N}\left(|y| \lambda^{1 / 2}\right) d y(\lambda)
$$

is continuous on $G \times G$ by the Lebesgue dominated convergence theorem, since $1 \leq \Omega_{N}(i r) \leq \Omega_{N}\left(i r_{1}\right)$ if $0 \leq r \leq r_{1}$.

If we choose a sequence $\left(\phi_{n}\right)$ in $\mathfrak{D}^{\#}(G)$ as above for $x_{0}=0$ and substitute $\phi_{n}$ for $\psi$ in (18) and let $n \rightarrow \infty$, we obtain

$$
\int_{G} f(x) \phi(x) d x=\int_{G} K(x, 0) \phi(x) d x
$$

for all $\phi \in \mathfrak{D}^{\sharp}(G) . K(x, 0)=\int_{-\infty}^{\infty} \Omega_{N}\left(|x| \lambda^{1 / 2}\right) d y(\lambda)$.

From (19) follows, by Proposition 1 and continuity of $f(x)$ and $K(x, 0)$ on $G$, that

$$
f(x)=\int_{-\infty}^{\infty} \Omega_{N}\left(|x| \lambda^{1 / 2}\right) d y(\lambda) \text { for all }|x|<a / 2 .
$$

To complete the proof we must show that (20) holds for all $|x|<a$. (If $a=\infty$ there is nothing to prove.)

Let

$$
F(x)=\int_{-\infty}^{\infty} \Omega_{N}\left(|x| \lambda^{1 / 2}\right) d y(\lambda) \text { for }|x|<a .
$$


The integral exists for $|x|<a$. This is seen as follows:

Using the formula [21, p. 367],

$$
\Omega_{N}(z u) \Omega_{N}(z v)=C_{N} \int_{0}^{\pi} \Omega_{N}\left(z\left(u^{2}+v^{2}-2 u v \cos \theta\right)^{1 / 2}\right)(\sin \theta)^{N-2} d \theta,
$$

where $u, v \in R, z$ complex and $C_{N}=\Gamma(N / 2)\left[\Gamma((N-1) / 2) \pi^{1 / 2}\right]^{-1}$, we have, for $0<$ $|x|<a / 2$,

$$
\begin{aligned}
K(x, x) & =\int_{-\infty}^{\infty}\left(\Omega_{N}\left(|x| \lambda^{1 / 2}\right)\right)^{2} d y(\lambda) \\
& =C_{N} \int_{-\infty}^{\infty} \int_{0}^{\pi} \Omega_{N}\left(\lambda^{1 / 2}|x|(2-2 \cos \theta)^{1 / 2}\right)(\sin \theta)^{N-2} d \theta d y(\lambda)
\end{aligned}
$$

from which follows that

$$
\int_{-\infty}^{0} \int_{0}^{\pi} \Omega_{N}\left(\lambda^{1 / 2}|x|(2-2 \cos \theta)^{1 / 2}\right)(\sin \theta)^{N-2} d \theta d y(\lambda)<\infty .
$$

(Note that the integrand is nonnegative.) Therefore,

$$
\int_{-\infty}^{\infty} \int_{0}^{\pi}\left|\Omega_{N}\left(\lambda^{1 / 2}|x|(2-2 \cos \theta)^{1 / 2}\right)\right|(\sin \theta)^{N-2} d \theta d \gamma(\lambda)<\infty .
$$

It follows by Fubini's theorem that

$$
\int_{-\infty}^{\infty}\left|\Omega_{N}\left(\lambda^{1 / 2}|x|(2-2 \cos \theta)^{1 / 2}\right)\right| d \gamma(\lambda)<\infty
$$

for almost all $0 \leq \theta \leq \pi$. But

$$
\int_{0}^{\infty}\left|\Omega_{N}\left(\lambda^{1 / 2}|x|(2-2 \cos \theta)^{1 / 2}\right)\right| d \gamma(\lambda)<\infty
$$

for all $0 \leq \theta \leq \pi$, and $\Omega_{N}\left(\lambda^{1 / 2}|x|(2-2 \cos \theta)^{1 / 2}\right) \leq \Omega_{N}\left(\lambda^{1 / 2}|x|\left(2-2 \cos \theta_{1}\right)^{1 / 2}\right)$ for $0 \leq \theta \leq \theta_{1} \leq \pi$ if $\lambda<0$. Therefore

$$
\int_{-\infty}^{\infty}\left|\Omega_{N}\left(\lambda^{1 / 2}|x|(2-2 \cos \theta)^{1 / 2}\right)\right| d \gamma(\lambda)<\infty
$$

for $0 \leq \theta<\pi$. Thus

$$
\int_{-\infty}^{\infty}\left|\Omega_{N}\left(\lambda^{1 / 2}|y|\right)\right| d y(\lambda)<\infty \text { for all }|y|<2|x|,
$$

and since $|x|<a / 2$ was arbitrary

$$
\int_{-\infty}^{\infty}\left|\Omega_{N}\left(|y| \lambda^{1 / 2}\right)\right| d y(\lambda)<\infty \quad \text { for all }|y|<a .
$$

From the preceding argument also follows, using the Lebesgue dominated convergence theorem, that $F(x)$ is continuous on $S_{N}(a)$.

Using the well-known formula $([15,(5)],[21$, p. 51])

$$
\Omega_{N}(z|t|)=\int_{|\xi|=1} e^{i z(\xi \cdot t)} d \sigma_{N}(\xi)
$$

where $z$ is any complex number, $t \in R^{N}$ and $\sigma_{N}$ the surface measure of the unit sphere $\left\{\xi \in R^{N}|| \xi \mid=1\right\}$ in $R^{N}$ normalized so that $\int d \sigma_{N}=1$, one verifies routinely that 
(23) $\int_{G} \int_{G} \Omega_{N}\left(|x-x| \lambda^{1 / 2}\right) \phi(x) \overline{\psi(y)} d x d y=\int_{G} \int_{G} \Omega_{N}\left(|x| \lambda^{1 / 2}\right) \Omega_{N}\left(|y| \lambda^{1 / 2}\right) \phi(x) \overline{\psi(y)} d x d y$ for all $\phi$ and $\psi$ in $\mathfrak{D}^{\#}(G)$. From this follows using Fubini's theorem that

$$
\int_{G} \int_{G} F(x-y) \phi(x) \overline{\psi(y)} d x d y=\int_{G} \int_{G} K(x, y) \phi(x) \overline{\psi(y)} d x d y
$$

for all $\phi$ and $\psi$ in $\mathfrak{D}^{\sharp}(G)$, and hence, by (18),

$$
\int_{G} \int_{G} f(x-y) \phi(x) \overline{\psi(y)} d x d y=\int_{G} \int_{G} F(x-y) \phi(x) \overline{\psi(y)} d x d y
$$

for all $\phi$ and $\psi$ in $\mathscr{D}^{\#}(G)$. Let $b(x)=f(x)-F(x)$ for $x \in S_{N}(a)$. Then $b$ is a continuous rotation invariant function and

$$
\int_{G} \int_{G} b(x-y) \phi(x) \psi(y) d x d y=0
$$

for all $\phi$ and $\psi$ in $\mathfrak{D}^{\#}(G)$. We assert that this implies that $b=0$, which will establish the "if" part of the theorem because of (21).

Suppose $\phi \in C_{0}^{\infty}(G)$ and $\psi \in \mathscr{D}^{\sharp}(G)$; then for every $\rho \in O(N)$,

$$
\begin{aligned}
\int_{G} \int_{G} b(x-y) \phi(x) \psi(y) d x d y=\int_{G} \int_{G} b(\rho(x-y)) \phi(x) \psi(y) d x d y \\
=\int_{G} \int_{G} b(x-y) \phi\left(\rho^{-1} x\right) \psi\left(\rho^{-1} y\right) d x d y=\int_{G} \int_{G} b(x-y) \phi\left(\rho^{-1} x\right) \psi(y) d x d y .
\end{aligned}
$$

Hence

$$
\begin{aligned}
\int_{G} \int_{G} b(x-y) \phi(x) \psi(y) d x d y & =\int_{O(N)} \int_{G} \int_{G} b(x-y) \phi\left(\rho^{-1} x\right) \psi(y) d x d y d \rho \\
& =\int_{G} \int_{G} b(x-y) \phi^{\sharp}(x) \psi(y) d x d y=0
\end{aligned}
$$

by Fubini's theorem and (24). Thus $\int_{G} \int_{G} b(x-y) \phi(x) \psi(y) d x d y=0$ for all $\phi \epsilon$ $C_{0}^{\infty}(G)$ and $\psi \in \mathscr{D}^{\sharp}(G)$. From this follows that

$$
\int_{G} b(x-y) \psi(y) d y=0 \text { for every } x \in G \text { and every } \psi \in \mathfrak{D}^{\sharp}(G) \text {. }
$$

We shall prove that (25) implies that $b(x)=0$ for all $|x|<a$. (Note that (25) trivially implies that $b(x)=0$ for $|x|<a / 2$.) For $N=1,(25)$ may be written in the form

$$
\int_{0}^{a / 2}[b(x+y)+b(x-y)] \psi(y) d y=0 \quad \text { for }|x|<a / 2 \text { and all } \psi \in \mathfrak{D}^{\sharp}(-a / 2, a / 2) \text {. }
$$

If $\left|x_{0}\right|<a / 2$, substitute in this equation for $\psi$ a function $\psi_{n} \in \mathscr{D}^{\sharp}(-a / 2, a / 2)$ which has the properties: $\psi_{n} \geq 0$, supp $\psi_{n} \subset\left\{x|| x|-| x_{0}||<1 / n\right\}$ and $\int \psi_{n} d x=1$. If we now let $n \rightarrow \infty$ we obtain that

$$
\left(b\left(x+x_{0}\right)+b\left(x-x_{0}\right)\right) / 2=0 \text { for }|x|<a / 2 .
$$

Therefore $b\left(2 x_{0}\right)+b(0)=0\left(\right.$ take $\left.x=x_{0}\right)$ and $b(0)=0$ (take $\left.x=x_{0}=0\right)$, whence $b\left(2 x_{0}\right)=0$. Thus $b(x)=0$ for all $|x|<a$. 
If $N \geq 2$, (25) may be rewritten using generalized spherical coordinates in the form $\left({ }^{2}\right)$

$$
\begin{aligned}
0= & \int_{|y|<a / 2} b(x-y) \psi(y) d y \\
& =\frac{2 \pi^{(N-1) / 2}}{\Gamma((N-1) / 2)} \int_{0}^{a / 2} \int_{0}^{\pi} H\left(\left(|x|^{2}-2|x| r \cos \theta+r^{2}\right)^{1 / 2}\right)(\sin \theta)^{N-2} \Psi(r) r^{N-1} d \theta d r
\end{aligned}
$$

for $|x|<a / 2$ and all $\psi \in \mathscr{D}^{\#}(G)$. Here $\psi(x)=\Psi(|x|)$ and $b(x)=H(|x|)$.

From this we conclude that $\int_{0}^{\pi} H\left(\left(t^{2}-2 t r \cos \theta+r^{2}\right)^{1 / 2}\right)(\sin \theta)^{N-2} d \theta=0$ for all $0 \leq t<a / 2$ and $0 \leq r<a / 2$. In particular, if we let $r=t$,

$$
\int_{0}^{\pi} H\left(t(2-2 \cos \theta)^{1 / 2}\right)(\sin \theta)^{N-2} d \theta=0 \text { for } 0 \leq t<a / 2
$$

If we make the substitution $x=(t / 2)(2-2 \cos \theta)^{1 / 2}, 0<t<a / 2$, we obtain (up to the factor $2^{\left.N-1 / t^{2(N-2)}\right)}$ the equation

$$
\int_{0}^{t} H(2 x) x^{N-2}\left(\left(t^{2}-x^{2}\right)^{1 / 2}\right)^{N-3} d x=0 \text { for } 0<t<a / 2,
$$

and hence for $0 \leq t<a / 2$.

If $N=3$, equation (26) shows that $\int_{0}^{t} H(2 x) x^{N-2} d x=0$ for all $0 \leq t<a / 2$ which implies that $H(2 x) x^{N-2}=0$ for $0 \leq x<a / 2$ whence $H(2 x)=0$ for $0<x<a / 2$.

If $N$ is an odd integer $>3$ then (26) implies that

$$
\begin{aligned}
\frac{d}{d t} \int_{0}^{t} H(2 x) x^{N-2}\left(\left(t^{2}-x^{2}\right)^{1 / 2}\right)^{N-3} d x & \\
& =(N-3) t \int_{0}^{t} H(2 x) x^{N-2}\left(\left(t^{2}-x^{2}\right)^{1 / 2}\right)^{N-5} d x=0
\end{aligned}
$$

for $0 \leq t<a / 2$ and therefore

$$
\int_{0}^{t} H(2 x) x^{N-2}\left(\left(t^{2}-x^{2}\right)^{1 / 2}\right)^{N-5} d x=0 \text { for } 0 \leq t<a / 2
$$

Continuing this way until the exponent of $\left(t^{2}-x^{2}\right)^{1 / 2}$ is zero we conclude that

$$
\int_{0}^{t} H(2 x) x^{N-2} d x=0 \quad \text { for } 0 \leq t<a / 2
$$

and hence as above $H(2 x)=0$ for $0 \leq x<a / 2$.

If $N$ is an even integer we first conclude using the above procedure that

$$
\int_{0}^{t} H(2 x) x^{N-2}\left(t^{2}-x^{2}\right)^{-1 / 2} d x=0 \text { for all } 0 \leq t<a / 2
$$

Multiplying both sides of (27) by $t$ and integrating we get

$$
\begin{gathered}
\int_{0}^{t} \int_{0}^{s} H(2 x) x^{N-2} \frac{s}{\left(s^{2}-x^{2}\right)^{1 / 2}} d x d s=\int_{0}^{t} \int_{x}^{t} H(2 x) x^{N-2} \frac{s}{\left(s^{2}-x^{2}\right)^{1 / 2}} d s d x \\
=\int_{0}^{t} H(2 x) x^{N-2}\left(t^{2}-x^{2}\right)^{1 / 2} d x=0 \quad \text { for } 0 \leq t<a / 2 .
\end{gathered}
$$

(2) Cf. (8) in the following article. 
Continuing this way we obtain the equacions

$$
\int_{0}^{t} H(2 x) x^{N-2}\left(\left(t^{2}-x^{2}\right)^{1 / 2}\right)^{2 n-1} d x=0 \text { for } 0 \leq t<a / 2 \text { and } n=0,1,2,3, \ldots
$$

If we now make the substitution $u=\left(t^{2}-x^{2}\right)^{1 / 2}$, equations (28) yield

$$
\begin{aligned}
& \int_{0}^{t} H\left(2\left(t^{2}-u^{2}\right)^{1 / 2}\right)\left(\left(t^{2}-u^{2}\right)^{1 / 2}\right)^{N-3} u^{2 n} d u=0 \\
& \text { for } 0 \leq t<a / 2 \text { and } n=0,1,2,3, \ldots .
\end{aligned}
$$

From this follows by the Stone-Weierstrass approximation theorem that, for fixed $0<t<a / 2$,

$$
\int_{0}^{t} H\left(2\left(t^{2}-u^{2}\right)^{1 / 2}\right)\left(\left(t^{2}-u^{2}\right)^{1 / 2}\right)^{N-3} g(u) d u=0
$$

for all continuous functions $g$ on $0 \leq u \leq t$. Therefore,

$$
H\left(2\left(t^{2}-u^{2}\right)^{1 / 2}\right)\left(\left(t^{2}-u^{2}\right)^{1 / 2}\right)^{N-3}=0 \text { for } 0 \leq u \leq t
$$

(note that $H\left(2\left(t^{2}-u^{2}\right)^{1 / 2}\right)\left(\left(t^{2}-u^{2}\right)^{1 / 2}\right)^{N-3}$ is continuous on $0 \leq u \leq t$ even if $N=2$ because $H(x)=0$ for $0 \leq x<a / 2)$; in particular, $H(2 t)=0$ for $0<t<a / 2$. We have thus proved that $H(2 t)=0$ for $0 \leq t<a / 2$ in all cases.

The "only if" part of the theorem is proved by direct computation. If

$$
f(x)=\int_{-\infty}^{\infty} \Omega_{N}\left(|x| \lambda^{1 / 2}\right) d y(\lambda) \text { for }|x|<a
$$

and $\phi \in \mathfrak{D}^{\#}(G)$, where $G=S_{N}(a / 2)$, then by (23),

$$
\int_{G} \int_{G} \Omega_{N}\left(|x-y| \lambda^{1 / 2}\right) \phi(x) \overline{\phi(y)} d x d y=\left|\int_{G} \Omega_{N}\left(|x| \lambda^{1 / 2}\right) \phi(x) d x\right|^{2} .
$$

Therefore,

$$
\begin{aligned}
\int_{G} \int_{G} f(x-y) \phi(x) \overline{\phi(y)} d x d y & =\int_{G} \int_{G} \int_{-\infty}^{\infty} \Omega_{N}\left(|x-y| \lambda^{1 / 2}\right) \phi(x) \overline{\phi(y)} d y(\lambda) d x d y \\
& =\int_{-\infty}^{\infty}\left|\int_{G} \Omega_{N}\left(|x| \lambda^{1 / 2}\right) \phi(x) d x\right|^{2} d y(\lambda) \geq 0
\end{aligned}
$$

by Fubini's theorem

$$
\begin{aligned}
& \left(\int_{G} \int_{G} \int_{-\infty}^{\infty}\left|\Omega_{N}\left(|x-y| \lambda^{1 / 2}\right) \phi(x) \overline{\phi(y)}\right| d y(\lambda) d x d y\right. \\
& \leq \int_{G} \int_{G} \int_{-\infty}^{0} \Omega_{N}\left(|x-y| \lambda^{1 / 2}\right)|\phi(x)||\phi(y)| d y(\lambda) d x d y \\
& \quad+\int_{G} \int_{G} \int_{0}^{\infty}\left|\Omega_{N}\left(|x-y| \lambda^{1 / 2}\right)\right||\phi(x)||\phi(y)| d y(\lambda) d x d y \\
& \leq \int_{G} \int_{G} f(x-y)|\phi(x)||\phi(y)| d x d y+2 \int_{G} \int_{G} \int_{0}^{\infty}\left|\Omega_{N}\left(|x-y| \lambda^{1 / 2}\right)\|\phi(x)\| \phi(y)\right| d y(\lambda) d x d y \\
& \left.<\infty \text { because }\left|\Omega_{N}\left(|x-y| \lambda^{1 / 2}\right)\|\phi(x)\| \phi(y)\right| \leq|\phi(x) \| \phi(y)| \text { for } \lambda \geq 0\right)
\end{aligned}
$$

and (30). This completes the proof.

Remarks. 1. A continuous function positive definite relative to the orthogonal group is necessarily real valued. This follows for example from the integral representation (11). 
2. It is clear from the proof of Theorem 2 that if we replace in the definition of a function $f$ positive definite relative to the orthogonal group continuity by local essential boundedness the conclusions of Theorem 2 hold with

$$
f(x)=\int_{-\infty}^{\infty} \Omega_{N}\left(|x| \lambda^{1 / 2}\right) d \gamma(\lambda) \text { for all }|x|<a
$$

replaced by

$$
f_{1}(x)=\int_{-\infty}^{\infty} \Omega_{N}\left(|x| \lambda^{1 / 2}\right) d y(\lambda) \text { exists for all }|x|<a
$$

and

$$
f(x)=f_{1}(x) \text { a.e. }
$$

3. The measure $\gamma$ in (11) may be chosen such that supp $\gamma \subset(-\infty, 0]$ if in addition to (10)

$$
\int s_{N}(a / 2) \int s_{N}(a / 2) f(x-y)(\Delta \phi)(x) \overline{\phi(y)} d x d y \geq 0
$$

for all radial $\phi \in C_{0}^{\infty}\left(S_{N}(a / 2)\right)$.

We recall that a continuous function $f$ on a ball $S_{N}(a)$ in $R^{N}$ is called (radial) exponentially convex (if it is radial and) if

$$
\sum_{i=1}^{n} \sum_{j=1}^{n} \alpha_{i} \bar{\alpha}_{j} f\left(x_{i}+x_{j}\right) \geq 0
$$

for all choices of complex numbers $\alpha_{1}, a_{2}, \cdots, \alpha_{n}$ and points $x_{1}, x_{2}, \cdots, x_{n}$ in $S_{N}(a / 2)$.

Condition (31) is clearly equivalent to

$$
\int S_{N^{(a / 2)}} \int_{S_{N}(a / 2)} f(x+y) \phi(x) \overline{\phi(y)} d x d y \geq 0
$$

for all $\phi$ and $\psi$ in $C_{0}^{\infty}\left(S_{N}(a / 2)\right)$. (It implies that $f$ is real valued.)

Now, a continuous function $f$ on a ball $S_{N}(a)$ in $R^{N}$ is radial exponentially convex [15, Proposition 2] if and only if there exists a bounded positive Radon measure $\gamma$ on $[0, \infty)$ such that

$$
f(x)=\int_{0}^{\infty} \Omega_{N}(i|x| t) d y(t) \text { for all } x \in S_{N}(a) .
$$

If we combine this result with Theorem 2 we obtain the following theorem.

Theorem 3. For a continuous function $f$ on a ball $S_{N}(a), 0<a \leq \infty$, in $R^{N}$ the following statements are equivalent:

1. $f$ is positive definite relative to the orthogonal group $O(N)$.

2. There exists a bounded positive Radon measure $\gamma$ on $R$ such that

$$
f(x)=\int_{-\infty}^{\infty} \Omega_{N}\left(|x| \lambda^{1 / 2}\right) d y(\lambda) \quad \text { for all } x \in S_{N}(a) .
$$


3. There exist bounded positive rotation invariant Radon measures $\mu_{1}$ and $\mu_{2}$ on $R^{N}$ such that $\int_{R^{N}} e^{x \cdot t} d \mu_{2}(t)<\infty$ for all $x \in S_{N}(a)$ and

$$
f(x)=\int_{R} N e^{i x \cdot t} d \mu_{1}(t)+\int_{R} N e^{x \cdot t} d \mu_{2}(t) \quad \text { for all } x \in S_{N}(a) .
$$

4. There exists a continuous radial positive definite function $f_{1}$ on $R^{N}$ and a continuous radial exponentially convex function $f_{2}$ on $S_{N}(a)$ such that

$$
f(x)=f_{1}(x)+f_{2}(x) \text { for all } x \in S_{N}(a) .
$$

Proof. $(1) \Leftrightarrow(2)$. This is the content of Theorem 2 .

$(2) \Rightarrow(4)$. Indeed, the equation

$$
f(x)=\int_{-\infty}^{\infty} \Omega_{N}\left(|x| \lambda^{1 / 2}\right) d y(\lambda) \text { for all } x \in S_{N}(a)
$$

may be rewritten in the form

$$
f(x)=\int_{0}^{\infty} \Omega_{N}(|x| t) d \gamma_{1}(t)+\int_{0}^{\infty} \Omega_{N}(i|x| t) d \gamma_{2}(t) \text { for all } x \in S_{N}(a),
$$

where $\gamma_{1}$ and $\gamma_{2}$ are bounded positive Radon measures on $[0, \infty)$ such that

$$
\int_{0}^{\infty} \Omega_{N}(i|x| t) d y_{2}(t)<\infty \text { for all } x \in S_{N}(a) .
$$

Let

$$
f_{1}(x)=\int_{0}^{\infty} \Omega_{N}(|x| t) d \gamma_{1}(t) \text { for all } x \in R^{N}
$$

and

$$
f_{2}(x)=\int_{0}^{\infty} \Omega_{N}(i|x| t) d \gamma_{2}(t) \text { for all } x \in S_{N}(a) ;
$$

then $f_{1}$ is a radial positive definite function on $R^{N}$ as one verifies immediately using (22). See [19, Theorem 1, p. 816]. $f_{2}$ is radial exponentially convex by the preceding discussion and $f(x)=f_{1}(x)+f_{2}(x)$ for all $|x|<a$.

$(4) \Rightarrow(3)$. This follows from the fact that every continuous positive definite function $f_{1}$ on $R^{N}$ and every continuous exponentially convex function $f_{1}$ on $S_{N}(a)$ has an integral representation of the form

$$
f_{1}(x)=\int_{R} e^{i x \cdot t} d \mu_{1}(t) \text { for all } x \in R^{N}
$$

and

$$
f_{2}(x)=\int_{R} e^{x \cdot t} d \mu_{2}(t) \text { for all } x \in S_{N}(a),
$$

respectively, where the Radon measures $\mu_{1}$ and $\mu_{2}$ are bounded, positive and unique. See [15]. Clearly $\mu_{1}$ and $\mu_{2}$ are rotation invariant if and only if $f_{1}$ and $f_{2}$ are radial, respectively.

(3) $\Rightarrow(1)$. This is obvious. 
Corollary 1. A continuous function on $R^{N}$ is radial positive definite if and only if it is positive definite relative to the orthogonal group and bounded.

Theorem 4. For a continuous function $f$ on a ball $S_{N}(a), 0<a \leq \infty$, in $R^{N}$ the following statements are equivalent:

1. $f$ is radial positive definite in $S_{N}(a)$.

2. There exists a radial positive definite function $f_{1}$ on $R^{N}$ sucb that $f(x)=$ $f_{1}(x)$ for all $x \in S_{N}(a)$.

3. $f$ is positive definite relative to the orthogonal group $O(N)$ and

$$
\int_{S_{N}(a / 2)} \int_{S_{N}(a / 2)} f(x-y)(-\Delta \phi)(x) \overline{\phi(y)} d x d y \geq 0
$$

for all radial $\phi \in C_{0}^{\infty}\left(S_{N}(a / 2)\right)$.

Proof. (1) $\Rightarrow(3)$. This was shown in the introduction.

(3) $\Rightarrow(2)$. By Theorem 2, $f(x)=\int_{0}^{\infty} \Omega_{N}\left(|x| \lambda^{1 / 2}\right) d y(\lambda)$ for all $x \in S_{N}(a)$ where $\gamma$ is a bounded positive Radon measure on $[0, \infty)$. Let $f_{1}(x)=\int_{0}^{\infty} \Omega_{N}\left(|x| \lambda^{1 / 2}\right) d y(\lambda)$ for $x \in R^{N}$. Then $f_{1}$ is a radial positive definite function in $R^{N}$ as noted above.

$(2) \Rightarrow 1$. Obvious.

Theorem 4'. For a continuous function $f$ on a ball $S_{N}(a), 0<a \leq \infty$, in $R^{N}$ the following statements are equivalent:

1. $f$ is radial exponentially convex in $S_{N}(a)$.

2. $f$ is positive definite relative to the orthogonal group and

$$
\int_{S_{N}(a / 2)} \int_{S_{N}(a / 2)} f(x-y)(\Delta \phi)(x) \overline{\phi(y)} d x d y \geq 0
$$

for all radial $\phi \in C_{0}^{\infty}\left(S_{N}(a / 2)\right)$.

Proof. If 1 holds then

$$
\begin{aligned}
\int S_{N}(a / 2) \int_{S_{N}(a / 2)} & f(x-y) \phi(x) \overline{\phi(y)} d x d y \\
= & \int_{S_{N}(a / 2)} \int_{S_{N}(a / 2)} f(x+y) \phi(x) \overline{\phi(y)} d x d y \geq 0
\end{aligned}
$$

for all radial $\phi \in C_{0}^{\infty}\left(S_{N}(a / 2)\right)$, anc therefore $f$ is positive definite relative to the orthogonal group $O(N)$. Furthermore,

$$
\begin{gathered}
\int_{S_{N}(a / 2)} \int S_{N^{(a / 2)}} f(x-y)(\Delta \phi)(x) \overline{\phi(y)} d x d y=\int_{S_{N^{(a / 2)}}} \int_{S_{N}(a / 2)} f(x+y)(\Delta \phi)(x) \overline{\phi(y)} d x d y \\
=\int_{S_{N}(a)} f(x)((\Delta \phi) * \bar{\phi})(x) d x=\int_{S_{N}(a)} f(x) \sum_{i=1}^{N^{\prime}}\left(\phi_{x_{i}} * \bar{\phi}_{x_{i}}\right)(x) d x \\
=\sum_{i=1}^{N} \int s_{N^{(a / 2)}} \int s_{N^{(a / 2)}} f(x+y) \phi_{x_{i}}(x) \overline{\phi_{x_{i}}(y)} d x d y \geq 0
\end{gathered}
$$

for all radial $\phi \in C_{0}^{\infty}\left(S_{N}(a / 2)\right)$. 
If 2 holds then by Theorem 2 and Remark 3 there exists a bounded positive Radon measure $y$ on $(-\infty, 0]$ such that

$$
f(x)=\int_{-\infty}^{0} \Omega_{N}\left(|x| \lambda^{1 / 2}\right) d y(\lambda) \text { for all } x \in S_{N}(a) .
$$

This equation may be rewritten in the form

$$
f(x)=\int_{0}^{\infty} \Omega_{N}(i|x| t) d \alpha(t) \text { for all } x \in S_{N}(a),
$$

where $\alpha$ is a bounded positive Radon measure on $[0, \infty)$. Hence $f$ is radial exponentially convex by [15, Proposition 2]. (This amounts to a simple computation using (22).)

Note. From the integral representation

$$
f(x)=\int_{0}^{\infty} \Omega_{N}(|x| t) d \mu(t)
$$

of a radial positive definite function on $R^{N}$ follows that $f$ is $[(N-1) / 2]$-times continuously differentiable [19, Lemma 4]. An exponentially convex function is analytic as seen from its integral representation. Therefore by 4 of Theorem $3 a$ function $f$ on $S_{N}(a)$ which is positive definite relative to the orthogonal group $O(N)$ is of class $C^{[(N-1) / 2]}$. Therefore if $N \geq 5$ condition 3 of Theorem 4 and condition 2 of Theorem $4^{\prime}$ are equivalent to

$-\Delta f$ is positive definite relative to $O(N)$ and $\Delta f$ is positive definite relative to $O(N)$, respectively.

Theorem 5. A real valued function $F$ defined on $0 \leq r<a(0<a \leq \infty)$ bas an integral representation of the form

$$
F(r)=\int_{-\infty}^{\infty} e^{r^{2} t} d \mu(t) \text { for all } 0 \leq r<a,
$$

where $\mu$ is a positive bounded Radon measure on $R$, if and only if $f_{N}(x)=$ $F(|x|), x \in S_{N}(a)$, is a positive definite function relative to the orthogonal group $O(N)$ in $S_{N}(a)$ for every $N=1,2, \ldots$.

Proof. If $F$ has an integral representation of the form (32) it may be rewritten in the form

$$
F(r)=\int_{0}^{\infty} e^{-r^{2} t} d \mu_{1}(t)+\int_{0}^{\infty} e^{r^{2} t} d \mu_{2}(t) \text { for all } 0 \leq r<a
$$

where $\mu_{1}$ and $\mu_{2}$ are bounded positive Radon measures on $[0, \infty)$ such that $\int_{0}^{\infty} e^{r^{2} t} d \mu_{2}(t)<\infty$ for all $0 \leq r<a$.

Let $G(r)=\int_{0}^{\infty} e^{-r^{2} t} d \mu_{1}(t)$ for $0 \leq r<\infty$ and $H(r)=\int_{0}^{\infty} e^{r^{2} t} d \mu_{2}(t)$ for $0 \leq$ $r<a$. Then $g_{N}(x)=G(|x|), x \in R^{N}$, is a radial positive definite function in $R^{N}$ for $N=1,2, \cdots$. See [19, Theorem 2, p. $817 \mid$ and $b_{N}(x)=H(|x|), x \in S_{N}(a)$, is an exponentially convex function in $S_{N}(a)$ for $N=1,2,3, \ldots$ See $[15$, Theorem 2]. Therefore 


$$
f_{N}(x)=F(|x|)=g_{N}(x)+b_{N}(x), \quad x \in S_{N}(a),
$$

is a positive definite function relative to the orthogonal group $O(N)$ in $S_{N}(a)$ for every $N=1,2, \cdots$, by 4 of Theorem 3 .

Conversely, if $f_{N}(x)=F(|x|), x \in S_{N}(a)$, is positive definite relative to the orthogonal group $O(N)$ for $N=1,2,3, \ldots$, then there exists by Theorem 2 for every $N$ a bounded positive Radon measure $\gamma_{N}$ on $R$ such that

$$
f_{N}(x)=\int_{-\infty}^{\infty} \Omega_{N}\left(|x| \lambda^{1 / 2}\right) d \gamma_{N}(\lambda)
$$

for all $x \in S_{N}(a)$. The proof now proceeds mutatis mutandis as in [15].

Equation (33) may be rewritten in the form

$$
f_{N}(x)=\int_{0}^{\infty} \Omega_{N}\left(|x|(2 N t)^{1 / 2}\right) d \alpha_{N}(t)+\int_{0}^{\infty} \Omega_{N}\left(i|x|(2 N t)^{1 / 2}\right) d \beta_{N}(t)
$$

for all $x \in S_{N}(a)$, or

$$
F(r)=\int_{0}^{\infty} \Omega_{N}\left(r(2 N t)^{1 / 2}\right) d \alpha_{N}(t)+\int_{0}^{\infty} \Omega_{N}\left(i r(2 N t)^{1 / 2}\right) d \beta_{N}(t)
$$

for all $0 \leq r<a$, where $\alpha_{N}$ and $\beta_{N}$ are bounded positive Radon measures on $[0, \infty)$ and $\beta_{N}$ is such that $\int_{0}^{\infty} \Omega_{N}\left(i r(2 N t)^{1 / 2}\right) d \beta_{N}(t)<\infty$ for all $0 \leq r<a$.

From (34) follows that

$$
F(0)=\left\|\alpha_{N}\right\|+\left\|\beta_{N}\right\|, \quad \text { whence }\left\|\alpha_{N}\right\| \leq F(0), \quad\left\|\beta_{N}\right\|<F(0)
$$

for all $N$. Hence there exists a subsequence $\left(\alpha_{N_{k}}\right)_{k \geq 1}\left(\left(\beta_{N_{k}}\right)_{k \geq 1}\right)$ of $\left(\alpha_{N}\right)\left(\left(\beta_{N}\right)\right)$ which converges to a bounded positive Radon measure $\alpha(\beta)$ on $[0, \infty)$ in the $w^{*}$-topology of the conjugate space of $C_{\infty}([0, \infty)$ ) (the space of complex valued continuous functions on $[0, \infty)$ vanishing at infinity).

Let $0 \leq r<a$, then for any $r<r^{\prime}<a$ and every positive number $B$,

$$
\begin{aligned}
\mid F(r) & -\int_{0}^{\infty} \Omega_{N}\left(r(2 N t)^{1 / 2}\right) d \alpha_{N}(t)-\int_{0}^{B} \Omega_{N}\left(i r(2 N t)^{1 / 2}\right) d \beta_{N}(t) \mid \\
& \leq \int_{B}^{\infty} \Omega_{N}\left(i r(2 N t)^{1 / 2}\right) d \beta_{N}(t) \\
& \leq \frac{\Omega_{N}\left(i r(2 N t)^{1 / 2}\right)}{\Omega_{N}\left(i r^{\prime}(2 N t)^{1 / 2}\right)} \int_{B}^{\infty} \Omega_{N}\left(i r^{\prime}(2 N t)^{1 / 2}\right) d \beta_{N}(t) \quad \text { by [15, Lemma 1] } \\
& \leq \frac{\Omega_{N}\left(i r(2 N t)^{1 / 2}\right)}{\Omega_{N}\left(i r^{\prime}(2 N t)^{1 / 2}\right)} \int_{0}^{\infty} \Omega_{N}\left(i r^{\prime}(2 N t)^{1 / 2}\right) d \beta_{N}(t) \\
& \leq \frac{\Omega_{N}\left(i r(2 N t)^{1 / 2}\right)}{\Omega_{N}\left(i r^{\prime}(2 N t)^{1 / 2}\right)}\left(F\left(r^{\prime}\right)+\left\|\alpha_{N}\right\|\right) \leq \frac{\Omega_{N}\left(i r(2 N t)^{1 / 2}\right)}{\Omega_{N}\left(i r^{\prime}(2 N t)^{1 / 2}\right)}\left(F\left(r^{\prime}\right)+F(0)\right) .
\end{aligned}
$$

Hence, if we choose $B$ to be a point at which $\beta$ is continuous (i.e. $\beta(\{B\})=0$ ), let 
$N=N_{k}$ and let $k \rightarrow \infty$, we obtain the inequality

$$
\left|F(r)-\int_{0}^{\infty} e^{-r^{2} t} d \alpha(t)-\int_{0}^{B} e^{r^{2} t} d \beta(t)\right| \leq e^{\left(r^{2}-r^{\prime 2}\right) B}\left(F\left(r^{\prime}\right)+F(0)\right)
$$

because $\lim _{N \rightarrow \infty} \Omega_{N}\left(z(2 N)^{1 / 2}\right)=e^{-z^{2}}$ uniformly on any compact subset of the complex plane (hence $\lim _{N \rightarrow \infty} \Omega_{N}\left(i r(2 N t)^{1 / 2}\right)=e^{r^{2} t}$ uniformly on $0 \leq t \leq B$ for fixed $r$ ) and $\lim _{N \rightarrow \infty} \Omega_{N}\left(r(2 N t)^{1 / 2}\right)=e^{r^{2} t}$ uniformly on $0 \leq t<\infty$. See [15] and [19, Lemma 3 , p. 8201 .

If we now let $B \rightarrow \infty$ in equation (36) through a sequence of points at which $\beta$ is continuous we obtain the equation $F(r)=\int_{0}^{\infty} e^{-r^{2} t} d \alpha(t)+\int_{0}^{\infty} e^{r^{2} t} d \beta(t)$ for every $0 \leq r<a$. This equation may be rewritten in the form (32). This completes the proof.

Since a continuous function $F$ on $0 \leq r<a(0<a \leq \infty)$ has an integral representation of the form

$$
F(r)=\int_{-\infty}^{\infty} e^{r t} d \mu(t) \text { for all } 0 \leq r<a,
$$

where $\mu$ is a bounded positive Radon measure on $R$, if and only if $F$ is exponentially convex on $0<r<a$ (see [15]), we obtain the following corollary.

Corollary 2. A continuous function $F$ on $0 \leq r<a(0<a \leq \infty)$ is exponentially convex on $0<r<a$ if and only if $f_{N}(x)=F\left(|x|^{2}\right), x \in S_{N}\left(a^{1 / 2}\right)$, is positive definite. relative to the orthogonal group $O(N)$ for every $N=1,2,3, \ldots$.

Corollary 2 is an extension of a theorem by I. J. Schoenberg [19, Theorem 3, p. 821] which states that a real valued function $F$ on $0 \leq r<\infty$ is completely monotonic (cf. loc. cit.) if and only if $f_{N}(x)=F\left(|x|^{2}\right), x \in R^{N}$, is radial positive definite for every $N=1,2,3, \ldots$.

To obtain Schoenberg's theorem we combine Corollary 1 with Corollary 2. We get

A continuous function $F$ on $0 \leq r<\infty$ is bounded and exponentially convex on $0<r<\infty$ if and only if $f_{N}(x)=F\left(|x|^{2}\right), x \in R^{N}$, is positive definite for every $N=1,2,3, \ldots$.

But a continuous exponentially convex function $F$ on $0<r<\infty$,

$$
F(r)=\int_{-\infty}^{\infty} e^{r t} d \mu(t) \text { for all } 0<r<\infty,
$$

where $\mu$ is a bounded positive Radon measure on $R$, is bounded if and only if supp $\mu \subset(-\infty, 0]$. From this follows that a continuous function $F$ on $0 \leq r<\infty$ is bounded and exponentially convex on $0<r<\infty$ if and only if $F$ bas an inte. gral representation of the form

$$
F(r)=\int_{0}^{\infty} e^{-r t} d \mu_{1}(t) \text { for all } 0 \leq r<\infty,
$$


where $\mu_{1}$ is a bounded positive Radon measure on $[0, \infty)$. Functions which have such an integral representation are precisely (by the classical theorem of Hausdorff-Bernstein and Widder, cf. [19]) functions which are completely monotonic on $0 \leq r<\infty$.

In summary the following holds for a continuous function $F$ on an interval $0 \leq r<a(0<a \leq \infty)$. For every $N=1,2,3, \cdots$ let $f_{N}(x)=F\left(|x|^{2}\right), x \in S_{N}\left(a^{1 / 2}\right)$. Then $F$ has a representation of the form

1. $F(r)=\int_{0}^{\infty} e^{-r t} d \mu(t)$ if and only if $f_{N}(x)$ is positive definite for every $N$. (For $a=\infty$ this was proved by Schoenberg [19, Theorem 2] and extended to $0<$ $a \leq \infty$ by Nussbaum [15, Theorem 1].)

2. $F(r)=\int_{0}^{\infty} e^{r t} d \mu(t)$ if and only if $f_{N}(x)$ is exponentially convex for every $N^{\circ}$ (Nussbaum [15, Theorem 21.)

3. $F(r)=\int_{-\infty}^{\infty} e^{r t} d \mu(t)$ if and only if $f_{N}(x)$ is positive definite relative to the orthogonal group $O(N)$ for every $N$. This is Theorem 5. (The measures $\mu$ in $1-3$ are all bounded positive Radon measures on the interval of integration indicated, respectively.)

6. Distributions positive definite relative to the orthogonal group.

Definition 2. Let $T$ be a distribution in a ball $S_{N}(a)=\left\{x \in R^{N}|| x \mid<a\right\}, 0<$ $a \leq \infty$, in $R^{N}$ and $G=S_{N}(a / 2)$.

(a) $T$ is said to be radial positive definite if it is rotation invariant and if $\left\langle T, \phi * \phi^{*}\right\rangle \geq 0$ for all $\phi \in \mathscr{D}(G)$.

(b) $T$ is said to be positive definite relative to the orthogonal group $O(N)$ if it is rotation invariant and if $\left\langle T, \phi * \phi^{*}\right\rangle \geq 0$ for all radial $\phi \in \mathscr{D}(G)$.

Here $\phi^{*}(x)=\overline{\phi(-x)}(=\overline{\phi(x)}$ if $\phi$ is radial $)$ and $*$ denotes convolution.

The following lemma has essentially been proved in the course of the proof of Theorem 2.

Lemma 1. The set of all elements $\{\phi * \psi\}$, where $\phi$ and $\psi$ are in $\mathfrak{D}^{\#}\left(S_{N}(a / 2)\right)$, is total in $\mathfrak{D}^{\sharp}\left(S_{N}(a)\right)$.

Proof. It is sufficient to show by the Hahn-Banach theorem that if $T \epsilon$ $\mathfrak{D}^{\#}\left(S_{N}(a)\right)^{\prime}$ and $\langle T, \phi * \psi\rangle=0$ for all $\phi$ and $\psi$ in $\mathscr{D}^{\#}\left(S_{N}(a / 2)\right)$ then $T=0$. By Proposition 1 this is equivalent to showing that if $T \in \mathscr{D}^{\circ H}\left(S_{N}(a)\right)$ and $\langle T, \phi * \psi\rangle=0$ for all $\phi$ and $\psi$ in $\mathfrak{D}^{\#}\left(S_{N}(a / 2)\right)$, then $\langle T, \phi\rangle=0$ for all $\phi \epsilon$ $\mathfrak{D}^{\#}\left(S_{N}(a)\right)$.

Let $T$ be a rotation invariant distribution in $S_{N}(a)$ such that $\langle T, \phi * \psi\rangle=0$ for all $\phi$ and $\psi$ in $\mathscr{D}^{\#}\left(S_{N}(a / 2)\right)$.

Fix $0<\delta<a / 2$ and for every $0<\epsilon<\delta$ let $\rho_{\epsilon}$ be a function in $\mathscr{D}^{\#}\left(S_{N}(a / 2)\right)$ such that $\rho_{\epsilon} \geq 0, \int \rho_{\epsilon} d x=1$ and supp $\rho_{\epsilon} \subset\left\{x \in R^{N}|| x \mid<\epsilon\right\}$. Then 


$$
\left\langle T,\left(\phi * \rho_{\epsilon}\right) *\left(\psi * \rho_{\epsilon}\right)\right\rangle=0
$$

for all $\phi$ and $\psi$ in $\mathscr{D}^{\#}\left(S_{N}((a / 2)-\delta)\right)$ and $0<\epsilon<\delta$. (37) may be rewritten in the form

$$
\left\langle T * \rho_{\epsilon} * \rho_{\epsilon}, \phi * \psi\right\rangle=0
$$

for all $\phi$ and $\psi$ in $\mathscr{D}^{\#}\left(S_{N}((a / 2)-\delta)\right)$. Now $T * \rho_{\epsilon} * \rho_{\epsilon}$ is a rotation invariant $C^{\infty}$. function when restricted to $S_{N}(a-2 \delta)$. (38) corresponds to equation (24). It follows as in the proof of Theorem 2 that

$$
T * \rho_{\epsilon} * \rho_{\epsilon}=0 \quad \text { in } S_{N}(a-2 \delta) .
$$

Thus if $\phi \in \mathscr{D}^{\sharp}\left(S_{N}(a)\right)$ and supp $\phi \subset S_{N}(a-2 \delta)$ then

$$
\left\langle T, \phi * \rho_{\epsilon} * \rho_{\epsilon}\right\rangle=\left\langle T * \rho_{\epsilon} * \rho_{\epsilon}, \phi\right\rangle=0 \text { for all } 0<\epsilon<\delta \text {. }
$$

From this follows since $\phi * \rho_{\epsilon} * \rho_{\epsilon} \rightarrow \phi$ in $\mathscr{D}^{\#}\left(S_{N}(a)\right)$ as $\epsilon \rightarrow 0$ that

$$
\langle T, \phi\rangle=0 \quad \text { for all } \phi \in \mathscr{D}^{\sharp}\left(S_{N}(a)\right)
$$

with supp $\phi \subset S_{N}(a-2 \delta)$. Since $0<\delta<a / 2$ was arbitrary it follows that $\langle T, \phi\rangle=0$ for all $\phi \in \mathfrak{D}^{\#}\left(S_{N}(a)\right)$.

The following theorem extends Theorem 2 to distributions positive definite relative to the orthogonal group.

Theorem 6. A distribution $T$ on a ball $S_{N}(a), 0<a \leq \infty$, in $R^{N}$ is positive definite relative to the orthogonal group $O(N)$ if and only if there exists a tempered positive Radon measure $\gamma$ on $R$ such that

$$
\langle T, \phi\rangle=\int_{-\infty}^{\infty} \tilde{\phi}\left(\lambda^{1 / 2}\right) d y(\lambda) \quad \text { for all } \phi \in \mathscr{D}\left(S_{N}(a)\right) \text {. }
$$

Here

$$
\tilde{\phi}(z)=\int_{S_{N}(a)} \Omega_{N}(z|x|) \phi(x) d x .
$$

The measure $\gamma$ may be chosen such that its support is contained in $[0, \infty)$ $((-\infty, 0])$ if in addition to $\left\langle T, \phi * \phi^{*}\right\rangle \geq 0$ for all $\phi \in \mathfrak{D}^{\sharp}\left(S_{N}(a / 2)\right)$

$$
\left\langle T,(-\Delta \phi) * \phi^{*}\right\rangle \geq 0 \quad(\langle T,(\Delta \phi) * \phi\rangle \geq 0)
$$

for all $\phi \in \mathfrak{D}^{\#}\left(S_{N}(a / 2)\right)$.

Proof. On $E=\mathscr{D}^{\#}(G), G=S_{N}(a / 2)$, we define a pseudo-inner product by setting

$$
(\phi, \psi)=\left\langle T, \phi * \psi^{*}\right\rangle \text { for } \phi \text { and } \psi \text { in } E \text {. }
$$

Proceeding exactly as in the proof of The orem 2 we conclude that there exists 
a positive Radon measure $\gamma$ on $R$ such that

$$
\left\langle T, \phi * \psi^{*}\right\rangle=\int_{-\infty}^{\infty} \tilde{\phi}\left(\lambda^{1 / 2}\right) \overline{\tilde{\psi}\left(\lambda^{1 / 2}\right)} d \gamma(\lambda)
$$

for all $\phi$ and $\psi$ in $E$ and that we may choose $\gamma$ in such a way that supp $\gamma \subset$ $[0, \infty)(\operatorname{supp} \phi \subset(-\infty, 0])$ if $(40)$ holds.

First we show that

$$
f_{2}(x)=\int_{\lambda<0} \Omega_{N}\left(|x| \lambda^{1 / 2}\right) d \gamma(\lambda)<\infty \text { for all }|x|<a .
$$

This is seen as follows: If $\phi \in \mathscr{D}^{\sharp}(G), \phi \geq 0$ and $\phi \neq 0$, then

$$
\int_{\lambda<0} \int_{G} \int_{G} \Omega_{N}\left(|x| \lambda^{1 / 2}\right) \Omega_{N}\left(|y| \lambda^{1 / 2}\right) \phi(x) \phi(y) d x d y d y(\lambda) \leq \int_{-\infty}^{\infty}\left|\tilde{\phi}\left(\lambda^{1 / 2}\right)\right|^{2} d y(\lambda)<\infty
$$

and hence by Fubini's theorem (and the fact that $\int_{G} \Omega_{N}\left(|x| \lambda^{1 / 2}\right) \phi(x) d x \geq 0$ for $\lambda<0$ )

$$
\int_{\lambda<0} \Omega_{N}\left(|x| \lambda^{1 / 2}\right) \Omega_{N}\left(|y| \lambda^{1 / 2}\right) d y(\lambda)<\infty
$$

for almost all $(x, y)$ in $G \times G$. From this follows since $t \rightarrow \Omega_{N}\left(t \lambda^{1 / 2}\right), t \geq 0$, is an increasing function for $\lambda<0$, that

$$
\int_{\lambda<0} \Omega_{N}\left(|x| \lambda^{1 / 2}\right) \Omega_{N}\left(|y| \lambda^{1 / 2}\right) d y(\lambda)<\infty
$$

for all $x$ and $y$ in $G$. Hence (if we argue as in Theorem 2 following (21)) $\int_{\lambda<0} \Omega_{N}\left(|x| \lambda^{1 / 2}\right) d y(\lambda)<\infty$ for all $|x|<a$. It follows-using the Lebesgue dominated convergence theorem-that

$$
f_{2}(x)=\int_{\lambda<0} \Omega_{N}\left(|x| \lambda^{1 / 2}\right) d \gamma(\lambda)
$$

is continuous on $|x|<a$. From this integral representation of $f_{2}$ it is seen that $f_{2}$ is radial exponentially convex on $S_{N}(a)$ and $\gamma((-\infty, 0))=f_{2}(0)<\infty$.

Next we show that there exists an integer $m \geq 0$ such that

$$
\int_{0}^{\infty} \frac{d \gamma(\lambda)}{(1+\lambda)^{N+m+1}}<\infty .
$$

This shows that $\gamma$ is tempered (since $\gamma((-\infty, 0))<\infty)$.

To prove this we construct a sequence of functions $\left(\omega_{n}\right)_{n \geq 1}$ which has the following properties:

1. $\omega_{n}=\phi_{n} * \psi_{n}$ where $\phi_{n}$ and $\psi_{n} \in \mathfrak{D}^{\sharp}(G)$.

2. $\hat{\omega}_{n}(t)=\hat{\phi}_{n}(t) \hat{\psi}_{n}(t) \geq 0$ for all $t \in R^{N}$.

3. $\lim _{n \rightarrow \infty} \hat{\omega}_{n}(t)=\hat{\omega}(t)$ exists for all $t \in R^{N}$ and $\hat{\omega}(t) \geq A /\left(1+|t|^{2}\right)^{N+m+1}$ for all $t \in R^{N}$, where $A>0$ and $m$ is a nonnegative integer.

4. $\left|\left\langle T, \omega_{n}\right\rangle\right| \leq 1$ for all $n$. 
(We follow the construction of a "barrier sequence" in [8, pp. 162-164]. The proof in loc. cit. contains some minor mistakes.)

Let $K=\left\{x \in R^{N}|| x \mid \leq r\right\}$, where $r$ is a fixed number $0<r<a$. Since $T$ is a continuous linear functional on $\mathcal{D}\left(S_{N}(a)\right)$ the restriction of $T$ to $\mathscr{D}_{K}\left(S_{N}(a)\right)$-the subspace of $\mathscr{D}\left(s_{N}(a)\right)$ consisting of all elements $\phi \in \mathscr{D}\left(s_{N}(a)\right)$ with supp $\phi \subset K-$ is continuous. Hence there exists an integer $m \geq 0$ and a number $\delta>0$ such that $|\langle T, \phi\rangle| \leq 1$ for all $\phi \in \mathscr{D}_{K}\left(S_{N}(a)\right)$ such that $\sup _{|p| \leq m ; x} \in K\left|D^{P} \phi(x)\right| \leq \delta$. Here $p=$ $\left(p_{1}, p_{2}, \cdots, p_{N}\right), p_{i}$ positive or zero integers, $|p|=p_{1}+p_{2}+\cdots+p_{N}$ and $D^{p}=$ $\left(\partial / \partial x_{i}\right)^{p} 1 \ldots\left(\partial / \partial x_{N}\right)^{p_{N}}$.

Let $\eta(x)$ be the function in $L^{1}\left(R^{N}\right)$ whose Fourier transform $\hat{\eta}(t)=$ $1 /\left(1+|t|^{2}\right)^{N+m+1}$ :

$$
\eta(x)=\frac{1}{(2 \pi)^{N}} \int_{R}^{N} \frac{e^{i x \cdot t}}{\left(1+|t|^{2}\right)^{N+m+1}} d t .
$$

Choose any function $\chi \in \mathscr{D}^{\#}\left(S_{N}(a)\right)$ with supp $\chi \subset K / 8=\left\{x \in R^{N}|| x \mid \leq r / 8\right\}$ and let

$$
\omega=B \eta\left(\chi * \chi^{*}\right)
$$

where $B>0$ is a constant to be determined later. Then supp $\omega \subset K / 4$ and

$$
\begin{aligned}
\hat{\omega}(t) & =B\left(\hat{\eta} *|\hat{\chi}|^{2}\right)(t)=B \int_{R}|\hat{\chi}(u)|^{2} \hat{\eta}(t-u) d u \\
& \geq B \int_{|u| \leq 1}|\hat{\chi}(u)|^{2} \frac{1}{\left(1+|t-u|^{2}\right)^{N+m+1}} d u \\
& \geq \frac{B}{\left(1+(|t|+1)^{2}\right)^{N+m+1}} \int_{|u| \leq 1}|\hat{\chi}(u)|^{2} d u \\
& =\frac{B C}{\left(1+(|t|+1)^{2}\right)^{N+m+1}}=B C\left(-\frac{\left(1+|t|^{2}\right)}{1+(|t|+1)^{2}}\right)^{N+m+1} \frac{1}{\left(1+|t|^{2}\right)^{N+m+1}} \\
& \geq \frac{B C^{\prime}}{\left(1+|t|^{2}\right)^{N+m+1}}
\end{aligned}
$$

for all $t \in R^{N}$, where $C^{\prime}=\inf _{t \in R^{N}}\left(\left(1+|t|^{2}\right) /\left(1+(|t|+1)^{2}\right)\right)^{N+m+1} C$. Thus

$$
\hat{\omega}(t) \geq A /\left(1+|t|^{2}\right)^{N+m+1} \text { for all } t \in R^{N}
$$

where $A=B C^{\prime}$.

From (43) we infer

(46) $\left|D^{p} \eta(x)\right| \leq \frac{1}{(2 \pi)^{N}} \int_{R} N \frac{|t||p| d t}{\left(1+|t|^{2}\right)^{N+m+1}} \leq M \quad$ for all $|p| \leq m$ and $x \in R^{N}$, 
where $M$ is a constant.

Now choose a function $\rho \in \mathscr{D}^{\sharp}\left(S_{N}(a)\right)$ with supp $\rho \subset K / 8, \int_{R} \rho d x=1$ and set $\rho_{n}(x)=n^{N} \rho(n x)$.

Let

$$
\phi_{n}=\rho_{n} * \rho_{n}^{*}, \quad \psi_{n}=\rho_{n} * \rho_{n}^{*} * \omega
$$

and

$$
\omega_{n}=\phi_{n} * \psi_{n}, \quad \text { for } n=1,2,3, \ldots
$$

Then conditions 1 and 2 are clearly satisfied. 3 is also satisfied because of (45) and $\hat{\omega}_{n}(t)=\hat{\omega}(t)\left|\hat{\rho}_{n}(t)\right|^{4}=\omega(t)|\hat{\rho}(t / n)|^{4} \rightarrow \hat{\omega}(t)|\hat{\rho}(0)|^{4}=\hat{\omega}(t)$ as $n \rightarrow \infty$ since $\hat{\rho}(0)=\int \rho(x) d x=1$. Finally, $\omega_{n} \in \mathfrak{D}_{K}\left(S_{N}(a)\right)$ and

$$
\begin{aligned}
\sup _{|p| \leq m ; x \in K}\left|D^{p} \omega_{n}(x)\right| & =\sup _{|p| \leq m ; x \in K}\left|\left(\left(D^{p} \omega\right) * \phi_{n} * \phi_{n}\right)(x)\right| \\
& \leq \sup _{|p|_{\leq m ; x} \in K}\left|D^{p} \omega(x)\right| \int_{R} N\left|\left(\phi_{n} * \phi_{n}\right)(x)\right| d x \\
& \leq \sup _{|p|_{\leq m ; x} \in K}\left|D^{p} \omega(x)\right|=B \sup _{|p| \leq m ; x \in K}\left|D^{p} \eta(x)\left(\chi * \chi^{*}\right)(x)\right|
\end{aligned}
$$

by (44). Hence-by (46)-if we choose $B$ sufficiently small

$$
\sup _{|p| \leq m ; x \in K}\left|D^{p} \omega_{n}(x)\right| \leq \delta
$$

for all $n$ and $\left|\left\langle T, \omega_{n}\right\rangle\right| \leq 1$ for all $n=1,2, \ldots$ Thus 4 is also satisfied.

Now we are ready to prove (42).

First we note that if $\phi \in \mathscr{D}\left(S_{N}(a)\right)$, then

$$
\begin{aligned}
\left\langle f_{2}, \phi\right\rangle & =\int_{R} f_{2}(x) \phi(x) d x=\int_{\lambda<0} \int_{R} \Omega_{N}\left(|x| \lambda^{1 / 2}\right) \phi(x) d x d y(\lambda) \\
& =\int_{\lambda<0} \tilde{\phi}\left(\lambda^{1 / 2}\right) d y(\lambda)
\end{aligned}
$$

by (41) and Fubini's theorem. Therefore

$$
\begin{aligned}
\int_{\lambda<0} \widetilde{\omega}_{n}\left(\lambda^{1 / 2}\right) d y(\lambda) & =\left\langle f_{2}, \omega_{n}\right\rangle=\left\langle f_{2}, \phi_{n} * \phi_{n} * \omega\right\rangle=\left\langle f_{2} * \check{\omega}, \phi_{n} * \phi_{n}\right\rangle \\
& =\left\langle f_{2} * \omega, \phi_{n} * \phi_{n}\right\rangle \rightarrow\left(f_{2} * \omega\right)(0)=\left\langle f_{2}, \omega\right\rangle \text { as } n \rightarrow \infty
\end{aligned}
$$

Here $\check{\omega}(x)=\omega(-x)$. Now

$$
\begin{aligned}
\int_{0}^{\infty} \tilde{\omega}_{n}\left(\lambda^{1 / 2}\right) d y(\lambda) & =\int_{-\infty}^{\infty} \tilde{\omega}_{n}\left(\lambda^{1 / 2}\right) d y(\lambda)-\left\langle f_{2}, \omega_{n}\right\rangle \\
& =\left\langle T, \omega_{n}\right\rangle-\left\langle f_{2}, \omega_{n}\right\rangle \leq 1+\left|\left\langle f_{2}, \omega_{n}\right\rangle\right|
\end{aligned}
$$

i.e.,

$$
\int_{0}^{\infty} \tilde{\omega}_{n}\left(\lambda^{1 / 2}\right) d y(\lambda) \leq 1+\left|\left\langle f_{2}, \omega_{n}\right\rangle\right| \text { for all } n
$$


Hence, if we let $n \rightarrow \infty$ it follows using Fatou's Lemma and 3 and (48), that $\int_{0}^{\infty}\left(A /(1+\lambda)^{N+m+1}\right) d \gamma(\lambda) \leq 1+\left|\left\langle f_{2}, \omega\right\rangle\right|<\infty$. This proves (42). From (42) follows that $\int_{0}^{\infty}\left|\mathcal{\phi}\left(\lambda^{1 / 2}\right)\right| d \gamma(\lambda)<\infty$ for all $\phi \in \mathscr{D}\left(R^{N}\right)$ and that $\langle S, \phi\rangle=\int_{0}^{\infty} \mathcal{\phi}\left(\lambda^{1 / 2}\right) d y(\lambda)$ for $\phi \in \mathscr{D}\left(R^{N}\right)$ defines a tempered distribution $S$ in $\mathscr{D}\left(R^{N}\right)$. Furthermore $S$ is obviously rotation invariant and

$$
\left\langle S, \phi * \phi^{*}\right\rangle=\int_{0}^{\infty}\left|\tilde{\phi}\left(\lambda^{1 / 2}\right)\right|^{2} d y(\lambda) \geq 0 \quad \text { for all } \phi \in \mathscr{D}\left(R^{N}\right) .
$$

Thus $S$ is a rotation invariant positive definite distribution in $R^{N}$.

Finally, if $\phi$ and $\psi$ are elements in $\mathcal{L}^{\sharp}(G)$ then

$$
\left\langle T, \phi * \psi^{*}\right\rangle=\int_{-\infty}^{\infty} \tilde{\phi}\left(\lambda^{1 / 2}\right) \overline{\tilde{\psi}\left(\lambda^{1 / 2}\right)} d \gamma(\lambda)=\left\langle S, \phi * \psi^{*}\right\rangle+\left\langle f_{2}, \phi * \psi^{*}\right\rangle
$$

and therefore by continuity and Lemma 1

$$
\langle T, \phi\rangle=\langle S, \phi\rangle+\left\langle f_{2}, \phi\right\rangle=\int_{-\infty}^{\infty} \tilde{\phi}\left(\lambda^{1 / 2}\right) d \gamma(\lambda)
$$

for all $\phi \in \mathfrak{D}^{\#}\left(S_{N}(a)\right)$. It follows from Proposition 1 that

$$
\langle T, \phi\rangle=\left\langle T, \phi^{\#}\right\rangle=\left\langle S, \phi^{\#}\right\rangle+\left\langle f_{2}, \phi^{\#}\right\rangle=\langle S, \phi\rangle+\left\langle f_{2}, \phi\right\rangle=\int_{-\infty}^{\infty} \tilde{\phi}\left(\lambda^{1 / 2}\right) d y(\lambda)
$$

for all $\phi \in \mathscr{D}\left(S_{N}(a)\right)$. The "if" part of Theorem 6 is obvious.

The following theorems are immediate corollaries of Theorem 6 and are analogous to Theorems 3, 4 and $4^{\prime}$. In fact they are extensions of these theorems, respectively.

Theorem 7. For a distribution $T$ on a ball $S_{N}(a), 0<a \leq \infty$, in $R^{N}$ the following statements are equivalent:

1. $T$ is positive definite relative to the orthogonal group.

2. There exists a radial positive definite distribution $S$ on $R^{N}$ and a radial exponentially convex function $f_{2}$ on $S_{N}(a)$ such that $T=S+f_{2}$ in $S_{N}(a)$.

Theorem 8. For a distribution $T$ on a ball $S_{N}(a), 0<a \leq \infty$, in $R^{N}$ the following statements are equivalent:

1. $T$ is radial positive definite in $S_{N}(a)$.

2. $T$ and $-\Delta T$ are both positive definite relative to the orthogonal group $O(N)$.

3. There exists a radial positive definite distribution $S$ on $R^{N}$ such that $T=S$ in $S_{N}(a)$.

The equivalence of 1 and 3 of the preceding theorem is an extension of Rudin's theorem [18] to distributions.

Theorem 8'. For a distribution $T$ on a ball $S_{N}(a), 0<a \leq \infty$, in $R^{N}$ the following statements are equivalent: 
1. $T$ is a radial exponentially convex function on $S_{N}(a)$.

2. $T$ and $\Delta T$ are both positive definite relative to the orthogonal group $O(N)$.

\section{REFERENCES}

1. Ju. M. BerezanskiY, Expansions in eigenfunctions of selfadjoint operators, "Naukova Dumka", Kiev, 1965; English transl., Transl. Math. Monographs, vol. 17, Amer. Math. Soc., Providence, R. I., 1968. MR 36 \#5768; \#5769.

2. S. Bochner, Vorlesungen über Fouriersche Integrale, Akad. Verlagsgesellschaft, Leipzig, 1932; English transl., Ann. of Math. Studies, no. 42, Princeton Univ. Press, Princeton, N. J., 1959. MR 21 \#5851.

3. A. P. Calderón and R. Pepinsky, On the phase of Fourier coefficients for positive real periodic functions, Computing Methods and the Phase Problem in X-ray Crystal Analysis, Department of Physics, Pennsylvania State College, State College, Pa., 1952, pp. 339-348.

4. A. Devinatz, On the extensions of positive definite functions, Acta Math. 102 (1959), 109-134. MR 22 \#875.

5. G. I. Éskin, A sufficient condition for the solvability of a multi-dimensional problem of moments, Dokl. Akad. Nauk SSSR 133 (1960), 540-543= Soviet Math. Dokl. 1 (1960), 895- 898. MR $22 \# 12394$.

6. L. (Maiding, Applications of the theory of direct integrals of Hilbert spaces to some integral and differential operators, The Institute for Fluid Dynamics and Appl. Math. Lecture Series, no. 11, University of Maryland, College Park, Md., 1954. MR 17, 159.

7. I. M. Gel'fand and A. G. Kostjučenko, Expansion in eigenfunctions of differential and other operators, Dokl. Akad. Nauk SSSR 103 (1955), 349-352. (Russian) MR 17, 388.

8. I. M. Gel'fand and N. Ja. Vilenkin, Generalized functions. Vol. 4: Some applications of harmonic analysis, Fizmatgiz, Moscow, 1961; English transl., Academic Press, New York, 1964. MR 26 \#4173; MR 30 \#4152.

9. A. Grothendieck, Produitstensoriels topologiques et espaces nucléaires, Mem. Amer. Math. Soc. No. 16 (1955). MR 17, 763.

10. L. Hörmander, Linear partial differential operators, Die Grundlehren der math. Wissenschaften, Band 116, Academic Press, New York; Springer-Verlag, Berlin, 1963. MR 28 \#4221.

11. M. G. Kreĭn, Sur le problème du prolongement des fonctions hermitiennes positives et continues, C. R. (Dokl.) Akad. Nauk URSS 26 (1940), 17-22. MR 2, 361.

12. - On a general method of decomposing Hermite-positive nuclei into elementary products, C. R. (Dokl.) Acad. Sci. URSS 53 (1946), 3-6. MR 8, 277.

13. B. Sz.-Nagy, Spektraldarstellung linearer Transformationen des Hilbertschen Raumes, Ergebnisse der Mathematik und ihrer Grenzgebiete, Band 5, no. 5, Springer, Berlin, 1942. MR 8, 276.

14. John von Neumann, On rings of operators. Reduction theory, Ann of Math (2) 50 (1949), 401-485. MR 10, 548.

15. A. E. Nussbaum, Radial exponentially convex functions, J. Analyse Math. 25 (1972), 277-288.

16. A. Pietsch, Nukleare lokal konvexe Räume, Akademie-Verlag, Berlin, 1965. MR $31 \# 6114$. 
17. W. Rudin, The extension problem for positive-definite functions, Illinois J. Math. 7 (1963), 532-539. MR 27 \#1779.

18. - An extension theorem for positive-definite functions, Duke Math. J. 37 (1970), 49-53. MR 40 \#7722.

19. I. J. Schoenberg, Metric spaces and completely monotone functions, Ann. of Math. 39 (1938), 811-841.

20. Fr. Trèves, Topological vector spaces, distributions and kernels, Academic Press, New York, 1967. MR 37 \#726.

21. G. N. Watson, A treatise on the theory of Bessel functions, 2nd ed., Cambridge Univ. Press, Cambridge; Macmillan, New York, 1944. MR 6, 64.

DEPARTMENT OF MATHEMATICS, WASHINGTON UNIVERSITY, ST. LOUIS, MISSOURI 63130 